\title{
Analysis of Option Trading Strategies Based on the Relation of Implied and Realized S\&P500 Volatilities
}

\author{
Alexander Brunhuemer, Gerhard Larcher*, Lukas Larcher \\ Johannes Kepler University Linz
}

\section{ARTICLE INFO}

Article history:

Received 07 April 2021

Revised 23 August 2021

Accepted 07 September 2021

Published 20 September 2021

\section{Keywords:}

Option-trading

S\&P500-index

Implied volatility

Realized volatility

\section{ABSTRACT}

In this paper, we examine the performance of certain short option trading strategies on the S\&P500 with backtesting based on historical option price data. Some of these strategies show significant outperformance in relation to the S\&P500 index. We seek to explain this outperformance by modeling the negative correlation between the S\&P500 and its implied volatility (given by the VIX) and through Monte Carlo simulation. We also provide free testing software and give an introduction to its use for readers interested in running further backtests on their own.

\section{Introduction}

This paper is a follow-up to the papers "Modeling and Performance of Certain Put-Write Strategies" (Larcher, Del Chicca, and Szölgyenyi, 2013) and "A Comparison of Different Families of Put-Write Option Strategies” (Del Chicca and Larcher, 2012). In those, we analyzed the historical performance of certain put-write option strategies based on the S\&P500 index during the time period from 1990 to 2010. Our aim was to support the assertion made by several authors (see for example (Day and Lewis, 1997), (Ungar and Moran, 2009) and (Santa-Clara and Saretto, 2009)) that put options on the S\&P500 with strikes in a certain out-of-the-money range seem to be systematically overpriced. Consequently, certain put-write strategies, when carried out in a suitable form, show systematic outperformance (for example, in relation to the underlying as a benchmark). The assertions above are, in a certain sense, equivalent to the following statement: "The implied volatility of options (in a certain range) systematically overestimates the subsequently realized volatility of the $S \& P 500$. ” The aims of the present paper are as follows:

\footnotetext{
* Corresponding author. 
- We will analyze the performance of the trading strategies studied in (Larcher, Del Chicca, and Szölgyenyi, 2013), yet this time for the period from 2010 to 2020, and we will analyze the stability of the results compared with the results from before 2010 .

- (Jackwerth and Rubinstein, 1996) and (Bakshi and Kapadia, 2003) also discuss consistently higher at-themoney implied volatilities compared to subsequently realized volatility, which would make selling options atthe-money attractive. Thus, we will analyze a further class of strategies which are based not only on short put positions but also on short call positions at-the-money. Collectively, we will refer to this type of strategies as "Lambda strategies" (since an essential component in these strategies is to combine short positions in a put and a call with the same strike and the payoff function of such a combination shows the form of the Greek lambda letter, $\Lambda$, see Figure 8). In practice this strategy is often referred to as "short straddle", however, as we really like the illustrative description, we use the term Lambda strategies. The theoretical investigation of this class of strategies is strongly motivated by the second author's highly successful real-market trading using such strategies in the period from 2012 to 2020.

- For both classes of trading strategies we provide free-to-use testing programs on www.lsqf.org. These programs enable readers to easily carry out many further tests on their own, for any choice of parameters. We will introduce these programs in this paper.

- Finally - in addition to the real-data backtests - we seek to explain the outperformance of these strategies using a simulation approach based on a modeled dependence of the VIX (the S\&P500 volatility index) on the S\&P500.

In section 2 we will first set out the theoretical background, introducing the basic structure of our trading strategies as well as the modeling and simulation approach, and then finish this part by drawing conclusions from this approach.

Section 3 presents the updated performance data for the put-write strategies from (Larcher, Del Chicca, and Szölgyenyi, 2013) and introduces the testing program for these strategies as provided on www.lsqf.org .

In section 4 we proceed with a comprehensive performance analysis of the Lambda strategies and explain the use of the corresponding software on www.lsqf.org.

Section 5 concludes the present paper with a short summary.

\section{Implied vs. realized volatility, dependence of VIX and S\&P500 and simulation of trading strategies}

What exactly do we mean by "over-estimation of the realized volatility by the implied volatility" and "dependence of the VIX and the S\&P500"?

In a certain sense, the value of the VIX gives the current average value of the implied volatilities of the S\&P500 based on S\&P500 options with about one month to expiration. More details on how the VIX is calculated and how to correctly interpret its value can be found in the corresponding CBOE whitepaper ${ }^{12}$ or in Chapter 5 of the monograph (Larcher, 2020). The value of the VIX at a certain time $t$ could, therefore, be seen as a forecast of the actually realized

\footnotetext{
${ }^{1}$ https://cdn.cboe.com/resources/vix/vixwhite.pdf
} 
volatility of the S\&P500 in the month following the time-point $t$, that is, in the time interval $\left[t, t+\frac{1}{12}\right]$. This time interval on average consists of about 20 trading days.

What do we mean by the "realized volatility of the S\&P500 in the time interval $\left[t, t+\frac{1}{12}\right]$ "? We will define it here as the historical volatility of the S\&P500 in that time interval calculated based on daily closing prices.

With this definition we can then compare the historical values of these two quantities. (Koopman et al., 2005) also compared and evaluated different methods to estimate the realized volatility, but we will further restrict ourselves to the case of the implied volatility (and the VIX as a shortcut to this) as this is the type of volatility which gives us information about the options market. In Figure 1 we see the performance of the S\&P500 from 2000 to 2020 (blue) and the relation between VIX (red) and the subsequently realized volatility (green) in this time period. In Figure 2 we see the difference between VIX and realized volatility in absolute values, while Figure 3 shows the difference between VIX and realized volatility as a percentage of the realized volatility value.

The picture we see is pretty clear: If we define "realized volatility" as we did above, then we are definitely looking at systematic over-estimation of realized volatility by the VIX. This fact is clearly illustrated especially in Figure 3 and was also already recognized in (Jackwerth and Rubinstein, 1996).

As regards the dependence between the S\&P500 and its volatility index VIX, we can usually observe the following phenomenon: Sudden changes in the S\&P500 are negatively related to corresponding changes in the VIX (i.e. the implied options volatility), e.g. see (French, Schwert, and Stambaugh, 1987). More casually speaking, an increase in the S\&P500 mostly leads to a decrease in the value of the VIX, whereas a decrease of the S\&P500 usually leads to a substantial increase of the VIX. To illustrate this fact, we show in Figure 4 - to give just one example - the performance of the S\&P500 compared with the performance of the VIX in the turbulent time interval from 24 August 2001 to 2 October 2001 (23 trading days).

We can clearly see the negative correlation between the two graphs. It is tempting, of course, to model this dependence of the VIX on the S\&P500. Some attempts suggest the following approach: Let us consider a time interval $[0, T]$, and for arbitrary $t \in[0, T]$ denote by $S_{t}$ the value of the S\&P500 at time $t$ and by $\sigma_{t}$ the value of the VIX at time $t$. It turns out that for "not too large values of T" (say, up to one month) a dependence of the form $\sigma_{t}=\sigma_{0}$. $\left(\frac{S_{0}}{S_{t}}\right)^{a}$, with $a=4$ comes surprisingly close to reality in most cases. To support this assertion, we illustrate the result of this modeling approach for the time period considered in Figure 4 (see Figure 5) and for some further randomly chosen time periods (see Figure 6) of 100 trading days (approximately five months) each. 

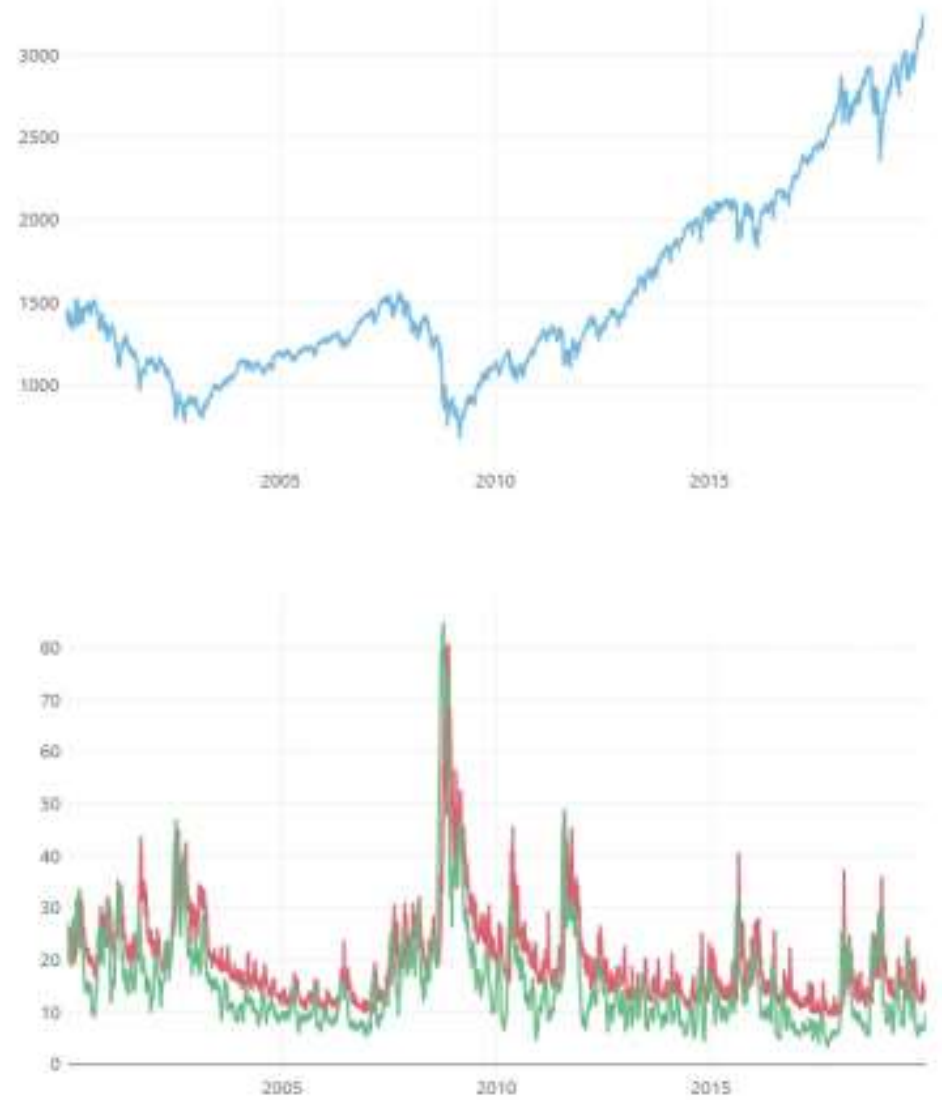

Figure 1. Comparison of SPX (blue line), VIX (red line), and realized volatility (green line) from January 2000 to December 2020

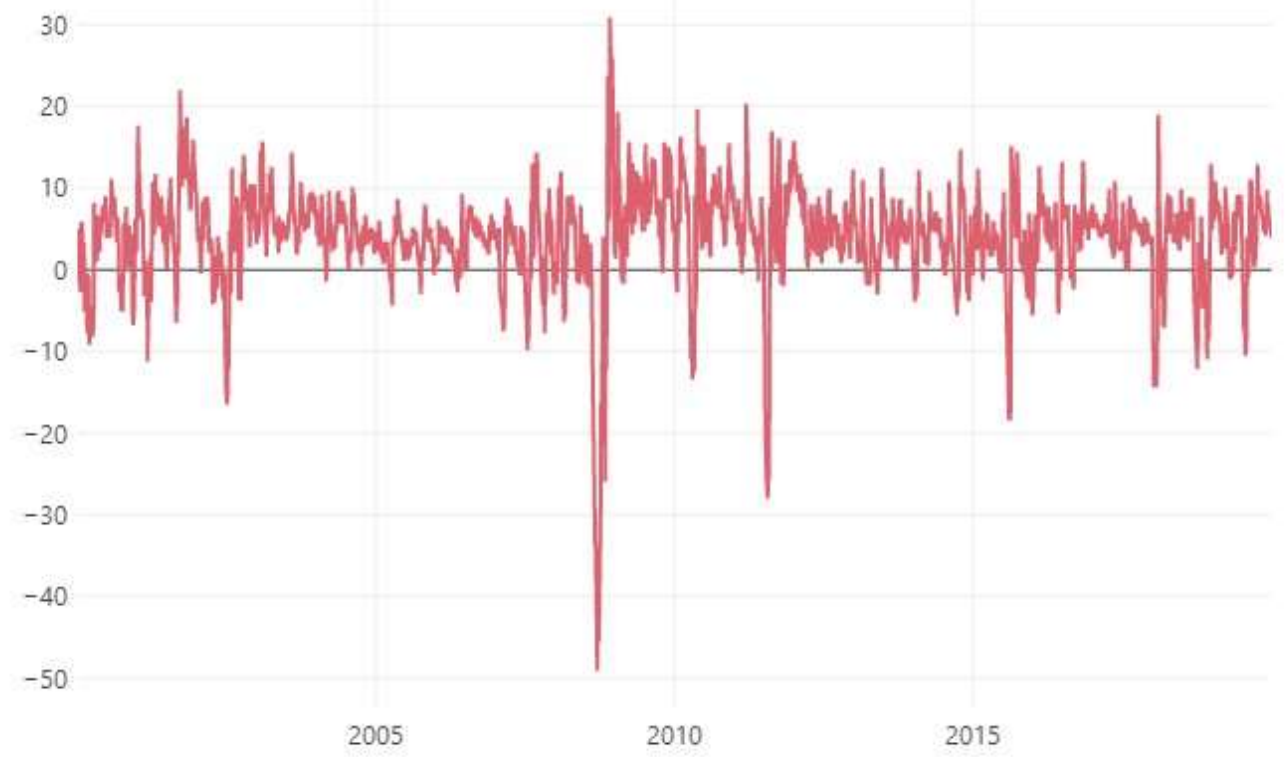

Figure 2. Difference between VIX and realized volatility in absolute values 


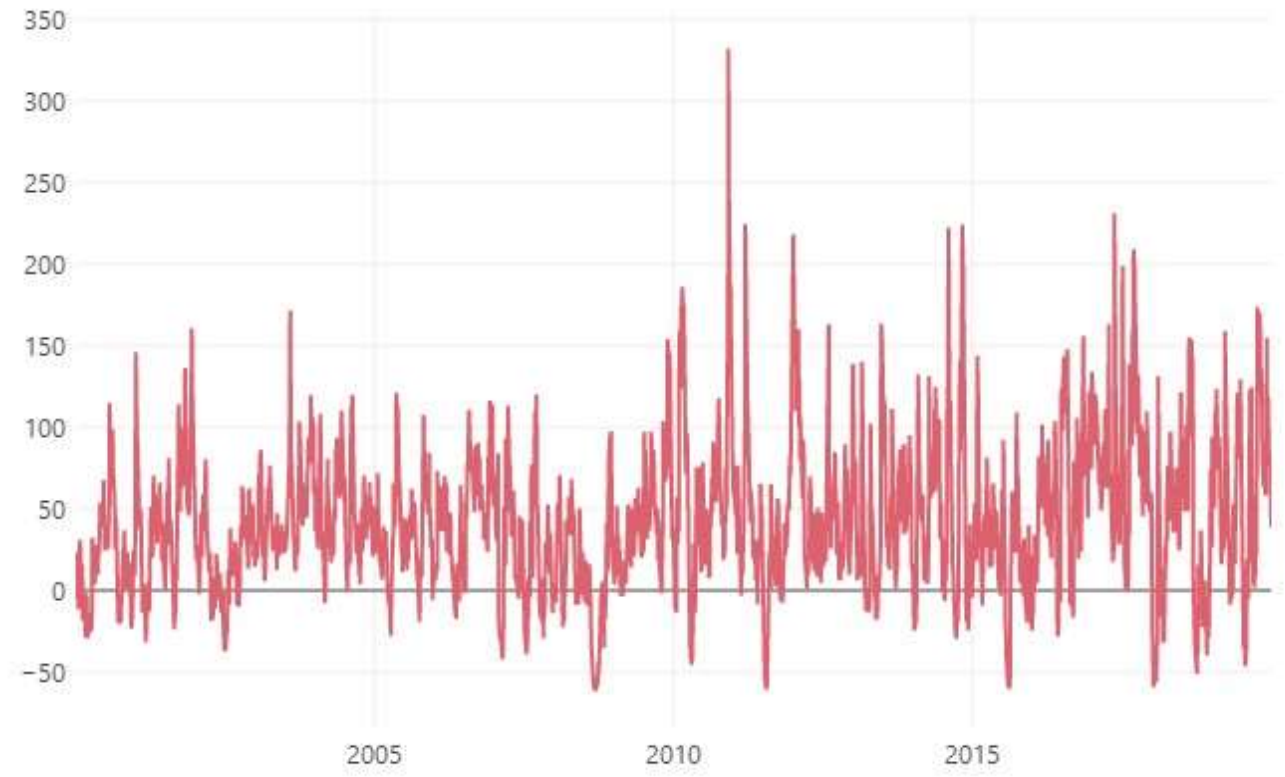

Figure 3. Difference between VIX and realized volatility as a percentage of the corresponding realized volatility

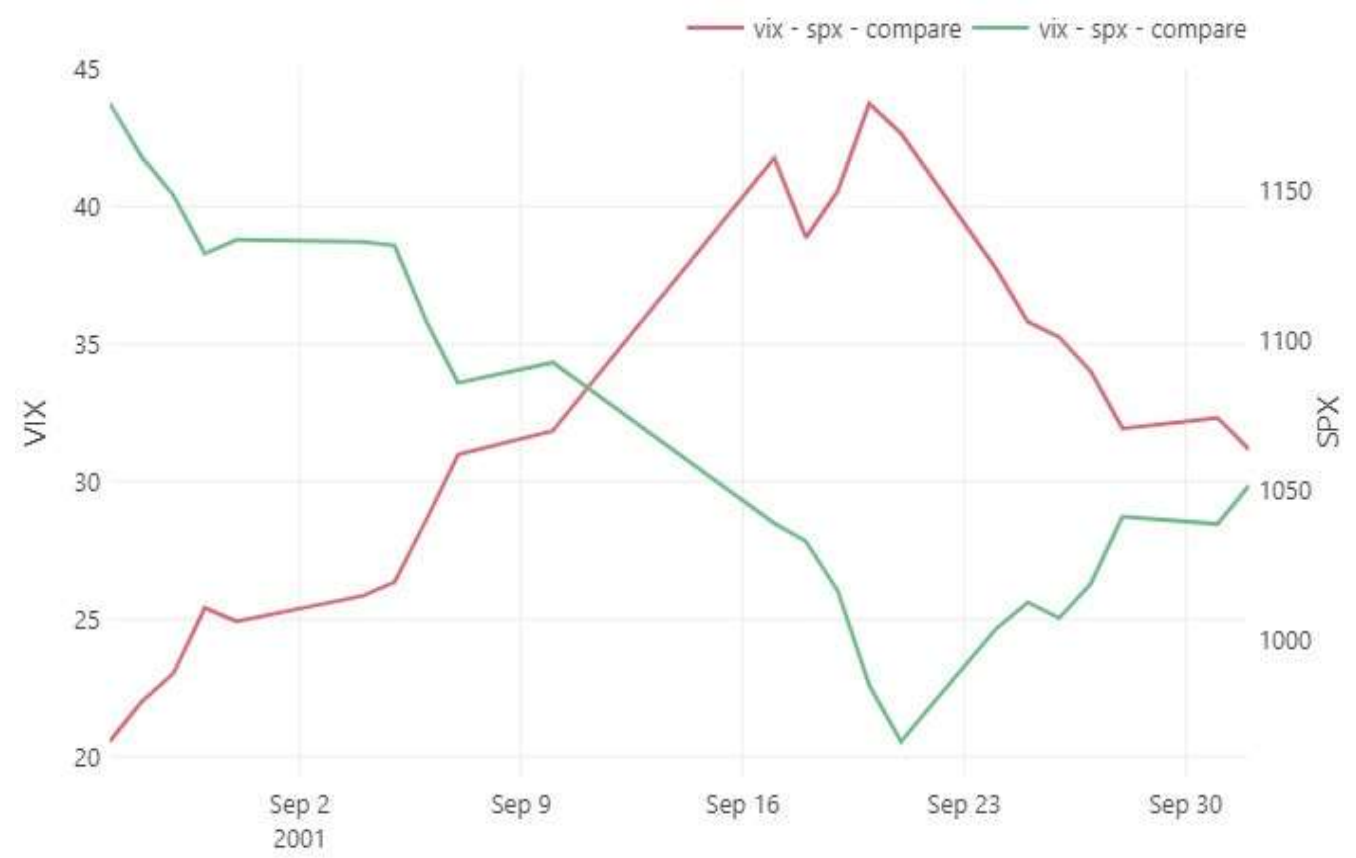

Figure 4. Comparison of VIX (red) and SPX (green) in the period from 2001-08-24 to 2001-10-02 


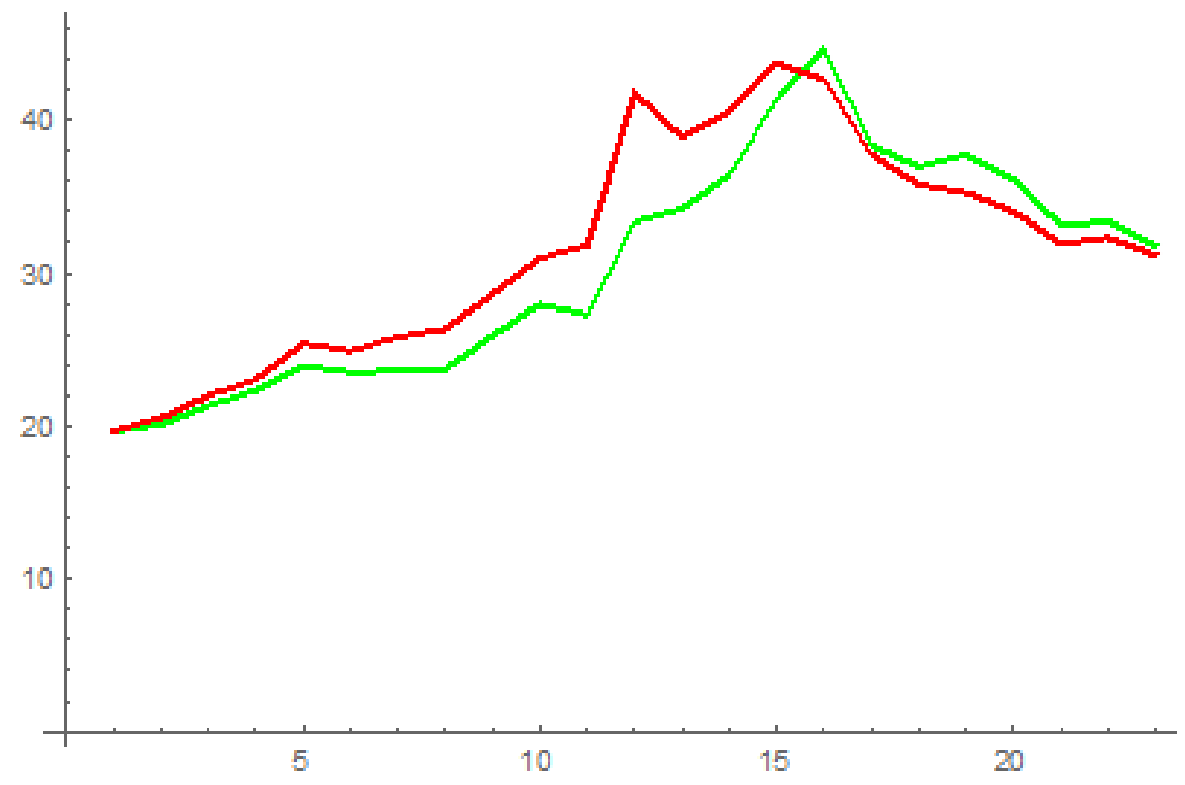

Figure 5. VIX (red) and $\sigma_{t}=\sigma_{0} \cdot\left(\frac{s_{0}}{s_{t}}\right)^{4}$ (green) for the time period from 2001-08-24 to 2001-10-2
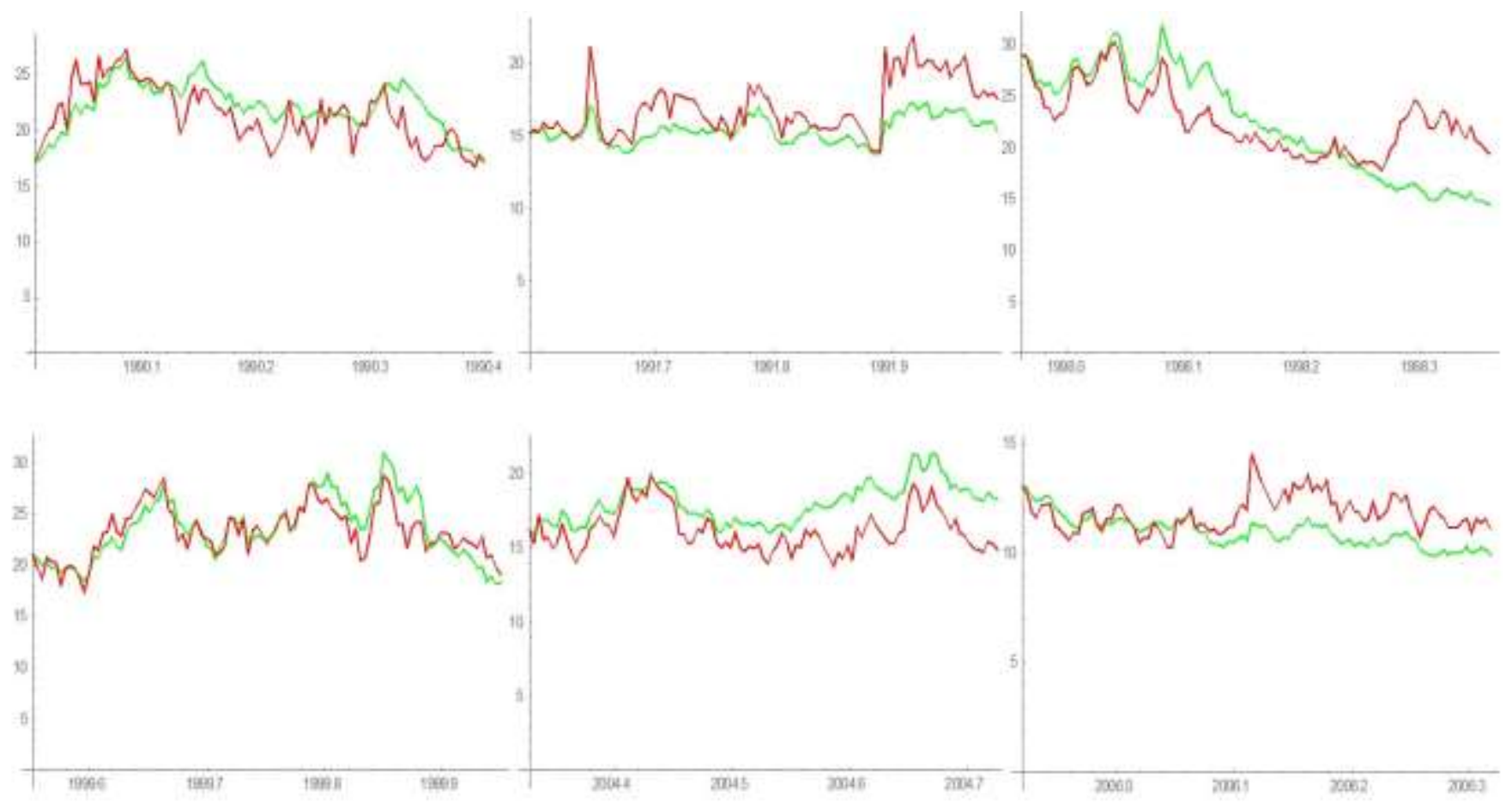

Figure 6. VIX (red) and $\sigma_{t}=\sigma_{0} \cdot\left(\frac{s_{0}}{s_{t}}\right)^{4}$ (green) for randomly chosen periods of 100 trading days

In Figure 5 we see a very good correlation between the real performance of the VIX and its modeling by $\sigma_{t}=\sigma_{0} \cdot\left(\frac{s_{0}}{s_{t}}\right)^{4}$. Figure 6 shows quite a good correlation in most cases, at least in the first quarter of the period, whereas after that, the quality of the modeling deteriorates in some cases. 
Based on this observation, we are going to run a simulation in the following for some of the trading strategies and will then investigate and backtest them in the subsequent parts, based on real historical option data. To this end we are going to introduce below the principal form of the two classes of trading strategies which we are focusing on here.

\section{The put-write strategy (principal structure)}

The basic idea of the put-write strategy is to repeatedly sell a put option at a pre-defined strike and make profit by gaining the premium. Whenever the underlying's value is staying "high enough" (and no exit strategy is executed beforehand), we gain profit by this trade. In the preceding paper (Larcher, Del Chicca, and Szölgyenyi, 2013), however, it was recognized that it makes sense to add a securing long put position at a lower strike, since margin requirements are clearer in this case and we can trade more contracts in that case. Hence, the rules we are following for our strategy are:

- We choose a fixed time period of length $T$ (e.g. 2 months, 1 month, one week, two trading days, ...).

- We trade SPX options with remaining time to expiration $T$ at times $0, T, 2 T, 3 T, \ldots$ (or with the shortest possible time to expiration larger than or equal to $T$, and with new trading upon expiration of these options).

- We always go short on put options with expiration $T$ (or approximately $T$ ) and strike $K_{1}$, and (in most cases) we go long on the same quantity of put options with the same expiration and with strike $K_{2}<K_{1}$. In a few cases we do not buy a long position but open a naked short position.

- So, upon entering the trade, we receive a positive premium of $M$ USD, which is given by the price of the short position minus the price of the long position.

- Our reference currency in all cases is the U.S. dollar (USD).

- The quantity of put option contracts to be traded may change and depends on the currently available investment amount and on the strategy we are running and investigating in our backtests.

- In some of the strategies the positions are held until expiration. Some of the strategies are equipped with an exit strategy, which means: All contracts are closed as soon as the losses from the two put positions (since the last trading day) exceed a certain pre-defined level.

- The strike price $K_{1}$ is chosen (depending on what strategy we are looking at) based on various parameters. It will always depend on the current value of the S\&P500 (at the trading date), in some cases it will also depend on the value of the VIX or on a certain historical volatility. In other cases, it will depend on the price of the put options in question.

- The strike $K_{2}$ is chosen as a function of $K_{1}$, e.g. of the form $K_{2}=K_{1}-L$ or $K_{2}=x \cdot K_{1}$, with a fixed strike distance $L$ or with $x$ as a fixed factor less than or equal to 1 .

- The trading assumptions in each case (bid/ask prices, the exact trading time, transaction costs, setting of a "buffer") are described in the section discussing the backtests of the put-write strategies. 


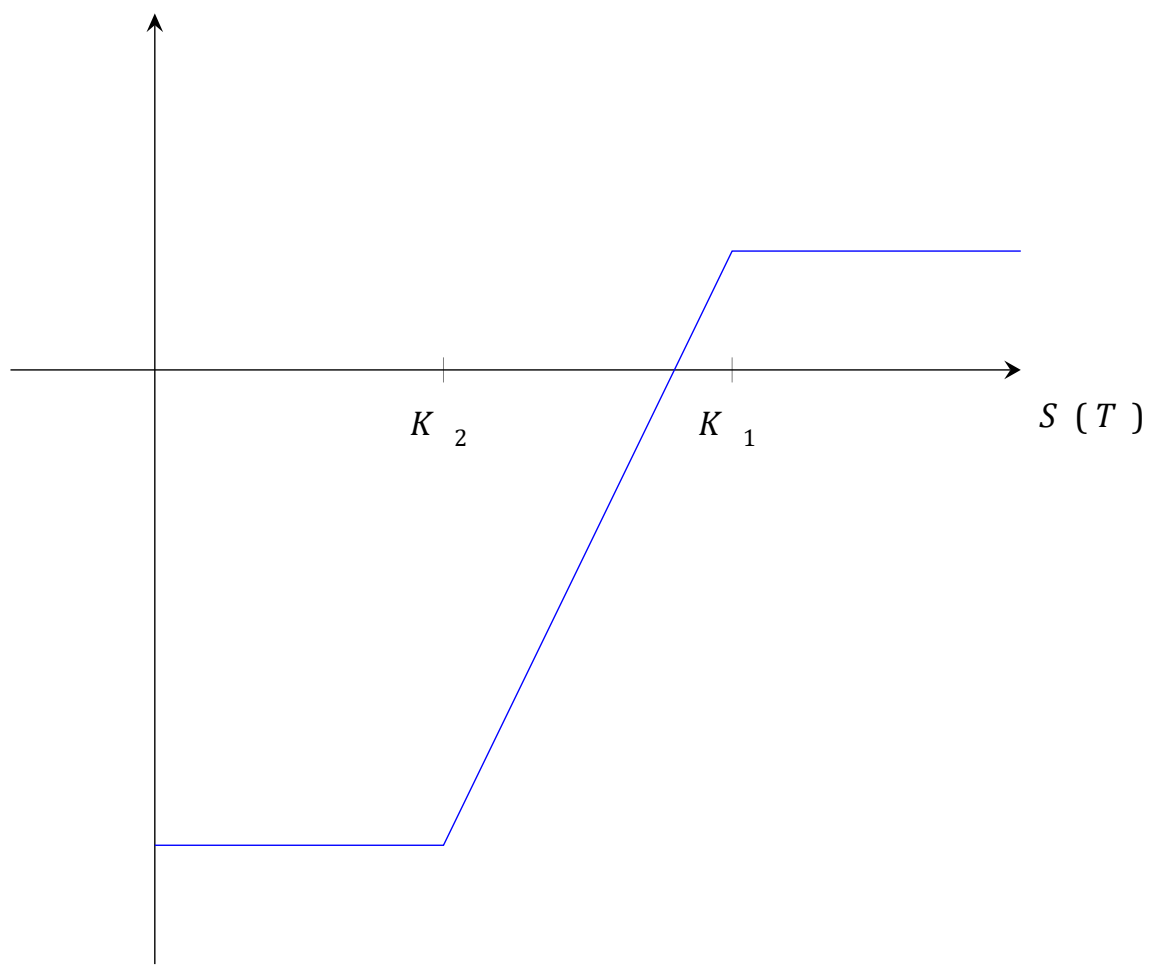

Figure 7. profit function of a put short with securing long positions

\section{The Lambda strategy (principal structure)}

The Lambda strategy is also based on selling options, but instead of only selling put options, we also sell call options, and we always do this at-the-money. By looking at this basic structure we see that calm markets with little volatility would work best to keep the gained premium. We could again open long positions to limit losses (which is also looked at in this paper), or one could react to changing market environments by trading the underlying asset (or futures of it for keeping trading costs low). The second suggestion will not be followed in this paper but should be kept in mind for further research. The strict rules we follow in our backtests are as follows:

- We choose a fixed time-period of length $T$ (e.g. 2 months, 1 month, one week, two trading days, ...)

- We trade SPX options with remaining time to expiration $T$ at times $0, T, 2 T, 3 T, \ldots$ (or with the shortest possible time to expiration larger than or equal to $T$ and with new trading upon expiration of these options).

- We always go short on put options with time to expiration $T$ (or approximately $T$ ) and strike $K_{1}$ as near the money as possible (i.e., with a strike as close as possible to the current value of the S\&P500), and we always go short on the same quantity of call options with the same expiration and the same strike $K_{1}$.

- In some cases we go long on the same quantity of put options with the same expiration and with a strike $K_{2}<$ $K_{1}$. In some cases we go long on the same quantity of call options with the same expiration and with a strike $K_{3}>K_{1}$. In some cases we go long on put options with strike $K_{2}<K_{1}$ as well as on call options with strike $K_{3}$ $>K_{1}$. In some cases we do not buy long positions but open only naked short positions.

- So, upon entering the trade, we receive a positive premium of $M$ USD, which is given by the price of the short positions minus the price of the long positions. 
- Our reference currency in all cases is the U.S. dollar (USD).

- The quantity of put option contracts to be traded may change and depends on the currently available investment amount and on the strategy we are running and investigating in our backtests.

- In some of the strategies the positions are held until expiration. Some of the strategies are equipped with an exit strategy, which means: All contracts are closed as soon as the losses from the call and put positions (since the last trading day) exceed a certain pre-defined level.

- Now - in contrast to the put-write strategies - it is the strikes $K_{2}$ and/or $K_{3}$ of the long positions that are chosen based on various parameters (depending on what strategy we are looking at). They will always depend on the current value of the S\&P500 (at the trading date); in some cases they will also depend on the value of the VIX or on a certain historical volatility, while in other cases they will depend on the prices of the put and/or call options in question.

- The trading assumptions in each case (bid/ask prices, the exact trading time, transaction costs, setting of a "buffer") are described in the section discussing the backtests of the Lambda strategies.

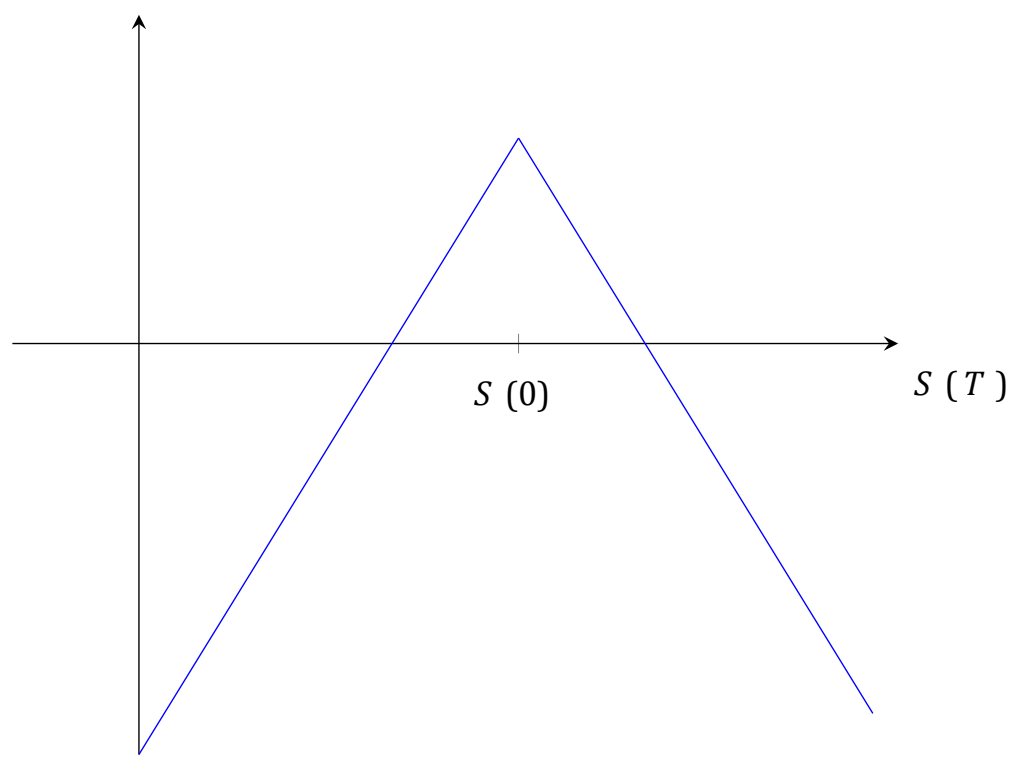

Figure 8. profit function of a pure lambda (short straddle) without securing long positions at the money

\section{Simulations of the strategies}

In the following we will carry out simulations of some basic examples of the above put-write and Lambda strategies. The reason for presenting these simulations is twofold:

- On the one hand we are going to illustrate that, if we work with the conventional Black-Scholes model and with fair Black-Scholes prices for the options, based on a constant volatility for the S\&P500 price and the same constant value for the implied option volatility (used for pricing the options), then these strategies are generally just zero-sum games (as is to be expected).

- On the other hand we are going to show that, if we model the implied volatility $\sigma_{t}$ (used for pricing the options) during the time interval $[0, T]$ not as a constant (equal to the fixed value $\sigma$ ), but for each single trading point $n T$ 
with the model $\sigma_{n T}=\sigma \cdot\left(\frac{s_{0}}{s_{n T}}\right)^{4}$ which we introduced above and which seems to reflect the reality better than the assumption of constant volatility, then in this case, these strategies usually show a clear outperformance.

This observation could serve as a further explanation of many results in the subsequent real-data backtests for the put-write strategies and the Lambda strategies, which also show a significant outperformance in many cases. For the simulation we use the following parameters and settings:

- We do not use long positions, i.e. we work with naked short positions.

- We study the performance of the strategies over a time interval of one year.

- The trading intervals we choose are $T=3$ days or $T=30$ days.

- At every trading date, we trade exactly one contract of the option combination (i.e. one short put contract for the put-write strategy and one short put contract together with one short call contract for the Lambda strategy).

- The simulations will be carried out for the "no exit strategy" cases and for exit strategies of the form "if the losses resulting from the open option positions exceed a certain absolute dollar amount at any point during their lifetime, then all contracts are to be closed".

- In the simulations we do not work with transaction costs, nor with bid/ask spreads, nor with any trading buffer.

- All tests will be carried out under the assumption of constant volatility over one trading year and dependence of the VIX on the S\&P500 of the form $\sigma_{t}=\sigma_{0} \cdot\left(\frac{S_{0}}{S_{t}}\right)^{4}$.

As our model for the S\&P500 we are going to use the one-dimensional risk-neutral Black-Scholes model.

That is, for the dynamics of the value $S_{t}$ of the S\&P500 at time $t$ we have $\mathrm{d} S_{t}=S_{t} r \cdot \mathrm{d} t+S_{t} \sigma \cdot \mathrm{d} W_{t}$, where $r$ is a constant risk-free interest rate on $[0, T], W_{t}$ is a standard Brownian motion, and $\sigma$ is the constant volatility over the trading year. Consequently, for all $0 \leq t_{1}<t_{2} \leq T$ we have

$$
S_{t_{2}}=S_{t_{1}} \cdot \exp \left(\left(r-\frac{\sigma^{2}}{2}\right)\left(t_{2}-t_{1}\right)+\sigma \sqrt{t_{2}-t_{1}} \cdot \omega\right)
$$

with a standard normally distributed random variable $\omega$. The initial value $S_{0}$ is assumed to be 3230 in all cases. If the entire trading period is given by $[0, N T]$ with trading times at $0, T, 2 T, \ldots,(N-1) T$, then we simulate the S\&P500 values $S_{T}, S_{2 T}, \ldots, S_{N T}$ using this model.

At each of these trading points $n T$ we calculate the Black-Scholes price of the options needed for the trade. As input for the volatility, we use either $\sigma_{n T}=\sigma \cdot\left(\frac{s_{0}}{S_{n T}}\right)^{4}$.

For every choice of parameters, we carry out 5,000 simulations and calculate the average annual return, the standard deviation of the 5,000 simulated returns, and the largest loss and the largest profit.

\section{Simulation of the put-write strategies}

In our first simulation experiment we consider the put-write strategy without long positions and with a short strike $K_{1}$, which is always chosen as a fixed percentage of the current S\&P500 value $S$ (at the moment of trading). For example, we choose $K_{1}=0.98 \cdot S$, or $K_{1}=0.9 \cdot S$, or $K_{1}=S$. 
In Figure 9 we show one sample path (blue) for such a simulation together with the corresponding trading dates ( $T=30$, hence 12 trades in one year) and the strikes (red, $K_{1}=0.9 \cdot S$ ) of the short positions.

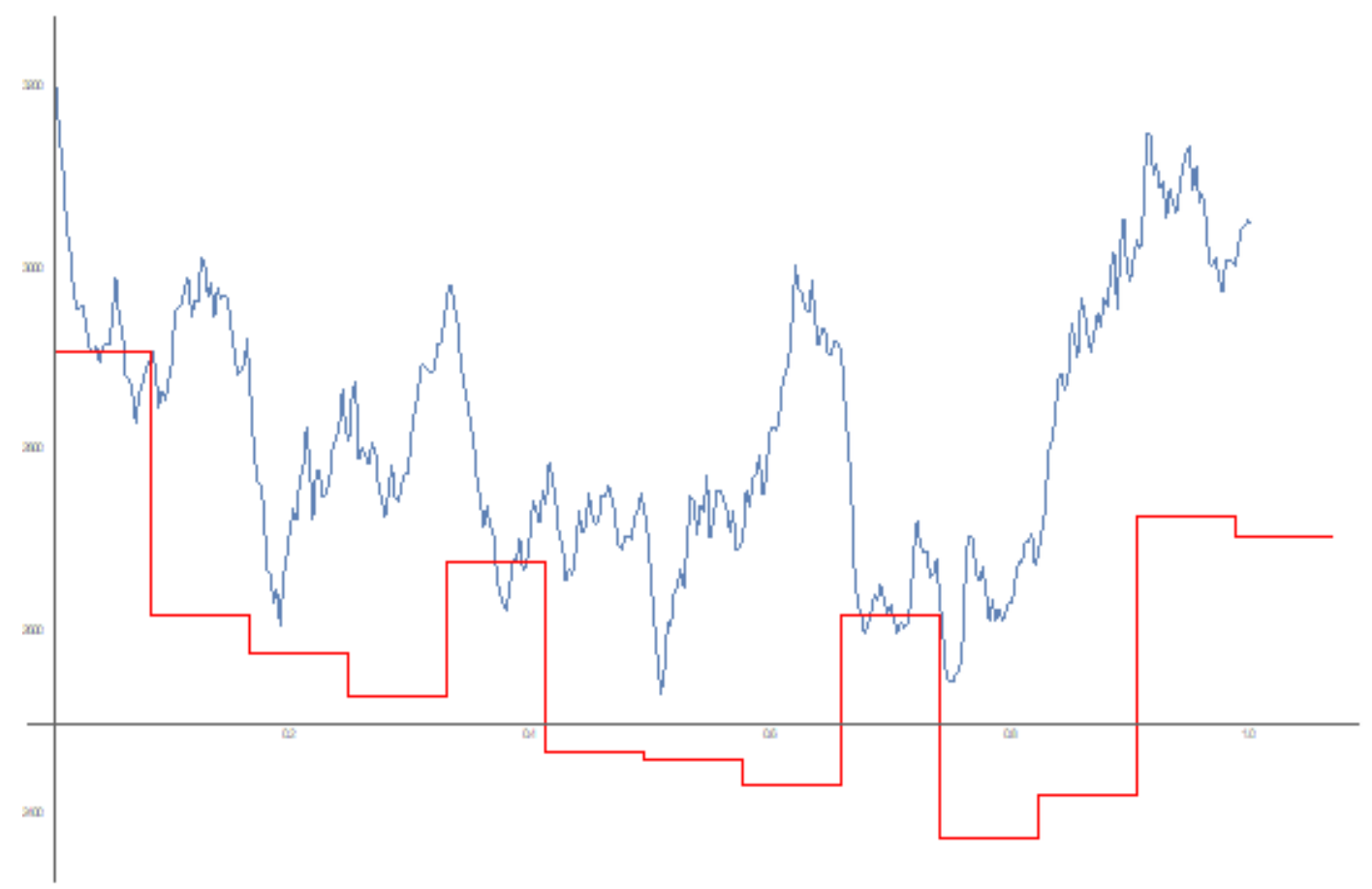

Figure 9. Sample path (blue) together with various strike choices for the strategy's different trading dates (red)

A small selection of the (many) results obtained is listed in the following Table 1. In most cases we rounded the results to multiples of 1,000 . 
Table 1. Selection of results of put-write simulations

\begin{tabular}{|c|c|c|c|c|c|c|c|c|c|}
\hline $\mathbf{r}$ & $\sigma$ & $\mathbf{a}$ & $\begin{array}{l}\text { T } \\
\text { days }\end{array}$ & $\mathbf{K}_{1}$ & Exit & average & $\begin{array}{l}\text { standard } \\
\text { deviation }\end{array}$ & Min & Max \\
\hline 0.01 & 0.2 & none & 30 & $0.98 \mathrm{~S}$ & none & -400 & 28.000 & -117.000 & 81.000 \\
\hline 0.06 & 0.2 & none & 30 & $0.98 \mathrm{~S}$ & none & -600 & 29.000 & -121.000 & 84.000 \\
\hline 0.01 & 0.6 & none & 30 & $0.98 \mathrm{~S}$ & none & -600 & 103.000 & -469.000 & 712.000 \\
\hline 0.01 & 0.2 & none & 3 & $0.98 \mathrm{~S}$ & none & 300 & 15.000 & -49.000 & 43.000 \\
\hline 0.01 & 0.2 & none & 30 & $0.90 \mathrm{~S}$ & none & -400 & 6.000 & 43.000 & 4.000 \\
\hline 0.01 & 0.2 & none & 30 & S & none & -500 & 36.000 & -98.000 & 109.000 \\
\hline 0.01 & 0.2 & 4 & 30 & $0.98 \mathrm{~S}$ & none & 9.400 & 29.000 & -95.000 & 122.000 \\
\hline 0.06 & 0.2 & 4 & 30 & $0.98 \mathrm{~S}$ & none & 3.200 & 28.000 & -95.000 & 161.000 \\
\hline 0 & 0.2 & 4 & 30 & $0.98 \mathrm{~S}$ & none & 11.900 & 30.000 & -96.000 & 157.000 \\
\hline 0.01 & 0.6 & 4 & 30 & $0.98 \mathrm{~S}$ & none & 199.000 & 339.000 & -519.000 & 1.170 .000 \\
\hline 0.01 & 0.2 & 4 & 3 & $0.98 \mathrm{~S}$ & none & 40.000 & 92.000 & -73.000 & 676.000 \\
\hline 0.01 & 0.2 & 4 & 30 & $0.90 \mathrm{~S}$ & none & 9.000 & 18.000 & -27.000 & 138.000 \\
\hline 0.01 & 0.2 & 4 & 3 & $0.90 \mathrm{~S}$ & none & 4.700 & 28.000 & 0 & 632.000 \\
\hline 0.01 & 0.2 & 4 & 30 & S & none & 8.900 & 29.000 & -82.000 & 105.000 \\
\hline 0.01 & 0.2 & 4 & 3 & S & none & 28.000 & 128.000 & -260.000 & 723.000 \\
\hline 0.01 & 0.2 & none & 30 & $0.98 \mathrm{~S}$ & -1.000 & 7.900 & 9.200 & -12.000 & 42.000 \\
\hline 0.01 & 0.2 & none & 30 & $0.98 \mathrm{~S}$ & -2.000 & 7.700 & 11.200 & -24.000 & 43.000 \\
\hline 0.01 & 0.2 & 4 & 30 & $0.98 \mathrm{~S}$ & -1.000 & 10.000 & 15.000 & -12.000 & 103.000 \\
\hline 0.01 & 0.2 & 4 & 30 & $0.98 \mathrm{~S}$ & -2.000 & 9.800 & 21.000 & -24.000 & 99.000 \\
\hline
\end{tabular}

Tendencies which can be deduced from our simulations (and which are supported by the sample values in Table 1) are:

- If we work with the assumption of constant implied volatility and no exit strategy, then the returns are around zero, regardless of the other parameters (zero-sum game).

- If we work with the assumption of constant implied volatility and an exit strategy with a rather tight loss limit, then the returns are slightly positive.

- If we work with the assumption of constant implied volatility and no exit strategy, then the standard deviation of the returns increases with the volatility of the underlying, and it also increases with increasing $K_{1}$. 
- If we work with the assumption of dependent implied volatility (following our model with parameter $a=4$ ) and no exit strategy, then the returns are systematically clearly positive.

- If we work with the assumption of dependent implied volatility (following our model with parameter $a=4$ ) and no exit strategy, then decreasing $r$ leads to increasing returns.

- If we work with the assumption of dependent implied volatility (following our model with parameter $a=4$ ) and no exit strategy, then increasing $\sigma$ leads to increasing returns.

- If we work with the assumption of dependent implied volatility (following our model with parameter $a=4$ ) and no exit strategy, then more frequent trading leads to increasing returns.

- If we work with the assumption of dependent implied volatility (following our model with parameter $a=4$ ) and no exit strategy, then a choice of $K_{1}$ of about $2 \%$ below $S$ yields significantly better results than a choice for $K_{1}$ equal to $S$ or about $10 \%$ below $S$.

- If we work with the assumption of dependent implied volatility (following our model with parameter $a=4$ ) and an exit strategy, then we get only slightly better results than in the case of constant implied volatility with an exit strategy.

\section{Simulation of the Lambda strategies}

The simulations for the Lambda strategy are carried out with the same principal basic model assumptions. In the simulations we do not work with long positions, i.e., we just short one put contract and one call contract with strike at the money at each trading date. We again provide a table (Table 2) with a small sample of simulation results for the Lambda strategy.

We can deduce similar findings as in the case of the put-write strategies:

- If we work with the assumption of constant implied volatility and no exit strategy, then the returns are around zero, regardless of the other parameters (zero-sum game).

- If we work with the assumption of constant implied volatility and an exit strategy with a rather tight loss limit, then the returns are slightly positive.

- If we work with the assumption of dependent implied volatility (following our model with parameter $a=4$ ) and no exit strategy, then the returns are mostly clearly positive.

- If we work with the assumption of dependent implied volatility (following our model with parameter $a=4$ ) and no exit strategy, then decreasing $r$ leads to increasing returns.

- If we work with the assumption of dependent implied volatility (following our model with parameter $a=4$ ) and no exit strategy, then increasing $\sigma$ leads to strongly increasing returns.

- If we work with the assumption of dependent implied volatility (following our model with parameter $a=4$ ) and an exit strategy, then we get only slightly better results than in the case of constant implied volatility with an exit strategy. An exit bound of about $-4,000$ USD seems to give the best results.

In general it seems that the Lambda strategy gives better average returns than the put-write strategies. 
Table 2. Selection of results of Lambda simulations

\begin{tabular}{|c|c|c|c|c|c|c|c|c|}
\hline $\mathbf{r}$ & $\sigma$ & $\mathbf{a}$ & $\begin{array}{l}\text { T } \\
\text { days }\end{array}$ & Exit & average & $\begin{array}{l}\text { standard } \\
\text { deviation }\end{array}$ & Min & Max \\
\hline 0.01 & 0.2 & none & 30 & none & -600 & 39.000 & -172.000 & 124.000 \\
\hline 0.06 & 0.2 & none & 30 & none & -1.500 & 40.000 & -172.000 & 115.000 \\
\hline 0 & 0.2 & none & 30 & none & 300 & 37.000 & -171.000 & 101.000 \\
\hline 0.01 & 0.6 & none & 30 & none & -600 & 123.000 & -567.000 & 314.000 \\
\hline 0.01 & 0.2 & none & 3 & none & -300 & 40.000 & -130.000 & 113.000 \\
\hline 0.01 & 0.2 & 4 & 30 & none & 15.000 & 93.000 & -340.000 & 447.000 \\
\hline 0.06 & 0.2 & 4 & 30 & none & -300 & 91.000 & -305.000 & 288.000 \\
\hline 0 & 0.2 & 4 & 30 & none & 22.000 & 93.000 & -250.000 & 326.000 \\
\hline 0.01 & 0.6 & 4 & 30 & none & 436.000 & 831.000 & -2.600 .000 & 2.600 .000 \\
\hline 0.01 & 0.2 & 4 & 3 & none & 69.000 & 302.000 & -560.000 & 1.570 .000 \\
\hline 0.01 & 0.6 & 4 & 3 & none & 2.428 .000 & 4.295.000 & -5.573 .000 & 18.556 .000 \\
\hline 0.01 & 0.2 & none & 30 & -1.000 & 11.000 & 13.000 & -12.000 & 46.000 \\
\hline 0.01 & 0.2 & 4 & 30 & -1.000 & 32.000 & 47.000 & -12.000 & 204.000 \\
\hline 0.01 & 0.2 & none & 30 & -2.000 & 11.000 & 20.000 & -24.000 & 75.000 \\
\hline 0.01 & 0.2 & 4 & 30 & -2.000 & 43.000 & 65.000 & -24.000 & 295.000 \\
\hline 0.01 & 0.2 & none & 30 & -4.000 & 11.000 & 22.000 & -48.000 & 88.000 \\
\hline 0.01 & 0.2 & 4 & 30 & -4.000 & 39.000 & 68.000 & -48.000 & 213.000 \\
\hline 0.01 & 0.2 & none & 30 & -5.000 & 15.900 & 25.000 & -60.000 & 73.000 \\
\hline 0.01 & 0.2 & 4 & 30 & -5.000 & 31.000 & 73.000 & -60.000 & 292.000 \\
\hline 0.01 & 0.6 & 4 & 30 & -4.000 & 222.000 & 342.000 & -48.000 & 1.915 .000 \\
\hline
\end{tabular}

Now we will turn to the real-data backtests of the two classes of strategies for the time period from 2010 to 2020 for the put-write strategies, and from 2012 to 2020 for the Lambda strategy. (The reason for the shorter testing interval for the Lambda strategy is that in this case, we also use the new type of weekly short-term options expiring on Monday, Wednesday and Friday of every week. These options are consistently available from 2012 onward only.) One goal of these backtests will be to establish whether the findings from the simulation experiments are generally borne out by the real-data backtests. 


\section{Real-data backtests for the put-write strategies}

Having presented the general idea of the put-write strategy in the previous section, we are now going to describe the detailed setting and execution of the strategy. We combine this with an explanation of the use of the accompanying software available on www.lsqf.org. The developed backtesting program provides an extensive interface allowing users to set up arbitrary combinations of parameters for our put-write strategies. The basic control parameters to be set by the user are

- the available initial investment capital $I$

- the period to be checked (start and end date)

- the duration of each period

- the formula for computing the short strike $K_{1}(s, v, h)$ depending on

- the underlying value $s$

- the value of the VIX as a percentage value $v$

- the annualized historical volatility $h$ of the previous 20 trading days

- the formula for computing the long strike $K_{2}(k)$ based on the short strike $k$

- the exit strategy

The reason for only allowing strategies hedged by a long position is to avoid uncertainties regarding potential margin calls in the case of naked short positions. By combining a short put option at strike $K_{1}$ with a long put option at strike $K_{2}<K_{1}$ the maximum loss for this combination is given by $K_{1}-K_{2}$, and therefore the required margin is also limited by that number.

In addition to the basic parameters above, the user can choose additional parameters to run more sophisticated strategies or consider real-market frictions.

\section{Basic parameter settings and execution of the strategy}

The following steps are repeated in a loop starting at the given start date until the end date is reached.

\section{Determining the upcoming trading period}

In the previous paper, a period always started on the third Friday of a given month and ended on the subsequent month's third Friday. For purposes of this approach the program on the website provides the "1-Month-Strategy" mode. In the last decade, however, the frequency of option expiry dates has increased enormously, allowing much more flexible choices of periods. For this reason the "Variable" mode was introduced, where users can choose a desired period length and the program will always search for options with expiration close to that chosen duration. In fact, it will choose the first expiration date greater than or equal to the desired period length. 


\section{Calculating the strikes}

The desired strikes for the short put and long put option, $\overline{K_{1}}$ and $\overline{K_{2}}$, are calculated based on the provided formulas. Except for the case of the "minimum premium" approach, which is introduced later, we always calculate the short strike $\overline{K_{1}}$ first. There are three different ways to define the short strike:

- $\overline{K_{1}}=x \cdot s$, where $x$ is a fixed factor and $s$ is the current price of the S\&P500. E.g. $\overline{K_{1}}=0.93 \cdot s$. This is the most basic choice of the short strike.

- $\overline{K_{1}}=(1-x \cdot h) \cdot s$, where $h$ is the S\&P500's historical annualized volatility of the last 20 trading days before the current trading date and $x$ is a constant factor. The idea behind this approach is to adapt the risk to the past volatility observed in the market. A higher volatility leads to a lower choice of the short strike. E.g. $\overline{K_{1}}=(1-0.4 \cdot h) \cdot s$

- The third approach is similar to the above in that $\overline{K_{1}}$ is calculated based on the anticipated market volatility, using the current VIX value: $\overline{K_{1}}=(1-x \cdot v) \cdot s$, where $v=\frac{V I X}{100}$. E.g. $\overline{K_{1}}=(1-0.4 \cdot v) \cdot s$

The desired long strike $\overline{K_{2}}$ is defined depending on the short strike. The two typical types are

- $\overline{K_{2}}=x \cdot k$, where $x$ is a fixed percentage and $k$ is the short strike $\overline{K_{1}}$. E.g. $\overline{K_{2}}=0.97 \cdot k$

- $\overline{K_{2}}=k-x$, where $x$ is a fixed distance to the short strike $k$. E.g. $\overline{K_{2}}=k-75$

It is required that $\overline{K_{2}}<\overline{K_{1}}$, otherwise the strategy won't make sense, since the cost of the long option would be higher than the premium earned from the short option. After defining the desired strikes $\overline{K_{1}}$ and $\overline{K_{2}}$, the options with the highest strike below the calculated strikes are determined and chosen for further execution of the strategy. With $K_{1}$ and $K_{2}$ we denote the actual strike of the chosen short and long put option.

\section{Determining the number of contracts to trade}

Before actually trading the short and long put contracts, we have to determine the number of contracts $N$ to trade. We are assuming contracts of 100 units in all our tests. Our goal is to use as much of our available investment capital as possible. The maximal loss for our option combination at the time of expiration is

$N \cdot 100 \cdot\left(K_{1}-K_{2}\right)$, therefore the required margin is also bounded by this value (for most brokers the required margin corresponds to exactly this value).

In addition to the available investment capital we also gain a certain premium $P$ at the beginning of the trade: $P=$ $100 \cdot N \cdot\left(P_{1}-P_{2}\right)$, where $P_{1}$ and $P_{2}$ are the respective prices of the short and the long put option at the beginning of the period. Therefore the following inequality has to hold:

$$
N \cdot 100\left(K_{1}-K_{2}\right) \leq I_{\text {curr }}+N \cdot 100\left(P_{1}-P_{2}\right)
$$

where $I_{\text {curr }}$ is the available capital in the current period. This leads to the following formula for the maximal number of contracts:

$$
N=\left\lfloor\frac{I_{c u r r}}{100\left(\left(K_{1}-K_{2}\right)-\left(P_{1}-P_{2}\right)\right)}\right\rfloor
$$


The prices $P_{1}$ and $P_{2}$ are calculated by averaging the last bid and ask price on the trading day on which we open the positions. Users can also choose a fixed distance to the averaged bid and ask price, e.g. they can state that 0.10 USD is to be added to the averaged bid/ask price when buying an option, or that 0.10 USD is to be subtracted when selling an option.

\section{Checking for execution of an exit strategy}

To limit the risk of a total loss of the investment, each strategy can also be equipped with an exit strategy. This exit strategy takes effect as soon as the aggregate losses of the open put positions exceed a chosen threshold and leads to an immediate closing of all open positions. This threshold is defined by the user as a percentage of the currently invested amount. Since we cannot use intraday option price data for each trading day we have to use an approximation to the worst intraday value of that combination of options. In our backtesting program this is handled in a slightly different way than in the previous paper, where the implied volatility was calculated at the end of the day and different option prices were calculated for this implied volatility and for different underlying values on this date. In the new program we look the short and long position's high values on each day and calculate for each day the worst value of the open positions as

$$
V=(\text { high value of long put }- \text { high value of short put }) \cdot N \cdot 100 \text {. }
$$

The reason for looking at this value is based on the following: The value of our open positions in the portfolio is usually negative since the lower strike of the long position implies a lower price for the corresponding option. Therefore, the best scenario for us is for this difference to be almost zero, or at least as small as possible. This is the case when the underlying asset's value is far greater than the strikes. On the other hand, the value $V$ decreases more and more the lower the underlying asset's value falls, which also leads to higher option prices. Additionally, it is reasonable to assume that the high values of the different put options occur at the same time, since they are all affected by the same underlying. Thus, it makes sense to compare the high values of the options to get the approximate "worst value" of our portfolio on a single day.

Thus, when the user enters an exit threshold of $y \%$ for the invested capital, the following check is run for each day:

$$
I_{\text {curr }}+\text { earned premium at period start date }+V<I_{\text {curr }}\left(1-\frac{y}{100}\right)
$$

If this inequality is satisfied, the exit strategy is activated and all open positions are closed. It is assumed that the trader closed all positions when the loss threshold was reached. To provide for delays in closing the options, users can enter an additional loss margin of $z \%$ (buffer), which is added to the exit threshold. The loss then adds up to $I_{\text {curr }} \cdot(y \%+z \%)$. In this case the strategy moves on to the next period. If the above inequality is never met during the lifetime of the positions, the strategy is considered successful and the final outcome for the current trading period is determined in the next step.

In the previous paper, exit strategies based on the underlying asset's value were also allowed, but because they did not work to a sufficiently reliable degree, this option was not implemented in the new program. 


\section{Determining the outcome}

The final outcome of the strategy is dependent on the underlying asset's closing value $S_{T}$ at expiration. The cases to consider are

- $S_{T}>K_{1}$ : Both option positions expire worthless, since the underlying value is above both strikes. Thus, the earned premium at the start of the period is kept as a profit.

- $K_{2} \leq S_{T} \leq K_{1}$ : The long position expires worthless, since $K_{2} \leq S_{T}$, however the short position has to be settled. Thus, $\left(K_{1}-S_{T}\right) \cdot N \cdot 100$ has to be paid.

- $S_{T}<K_{2}$ : Both option positions have to be settled since the underlying value is below $K_{1}$ and $K_{2}$. Thus, $\left(K_{1}-K_{2}\right) \cdot N \cdot 100$ has to be paid.

In each case the earned premium $N \cdot 100\left(P_{1}-P_{2}\right)$ has to be added in calculating the strategy's final profit.

\section{Additional parameters}

In addition to the fundamental strategy parameters, users can also choose additional parameters for more sophisticated variants of the strategy or simply to take existing market restrictions (e.g. transaction costs) into account.

\section{Transaction costs}

The transaction costs per contract can be provided and are subtracted accordingly. Where an exit strategy is activated, the transaction costs are considered twice.

Using the previous performance of the underlying asset in calculating the short strike In certain situations it seems useful, in calculating the short strike, to consider not only the current underlying value $S_{0}$ but also the underlying asset's price movement over the previous days. For example: Assume that, in the 5 trading days before the new period starts, the underlying price suddenly surges from 2000 to 2500. In this case the short strike would be calculated with the current underlying value of 2500 . The trader may, however, want to be a bit more cautious in such circumstances. For these scenarios the program offers an option to take the last $n$ trading days of the underlying into account, with $n$ $\in\{1, \ldots, 15\}$. The threshold value that is actually used for calculating the short strike can then be given based on the last underlying value $S_{0}$ and the minimal/maximal underlying value $S_{\min }$ and $S_{\max }$ in the last $n$ trading days. Typical inputs instead of $S_{0}$ for calculating the short strike could be of the form

- s_min

- s_max

- or even "(s_min $+\mathrm{s} \_$max $) / 2$ " is possible

We are not including this option in our upcoming tests, but it looks like an interesting variant for future research.

\section{Minimum-premium strategy}

Consider the idea that the calculation formula for the long strike $K_{2}$ is already given as a function of $K_{1}$. In this approach, we then choose for each period the pair $\left(K_{1}, K_{2}\right)$ in such a way that $K_{1}$ is the lowest value where the combination of put options with strikes $K_{1}$ and $K_{2}$ earns at least the specified percentage of the invested capital as 
premium. This means that for a user stating a premium of at least $3 \%$ for each trade and $K_{2}=0.95 \cdot K_{1}$, we would search for the lowest value of $K_{1}$, such that the combination of the short and the long position earns at least the stated $3 \%$ premium.

\section{No exit - capped risk strategy}

Exit strategies are a key concern in the definition of the put-write strategy. If thresholds are too strict, there will be more frequent losses, even if in hindsight, the strategy would have worked had no positions been closed. On the other hand, exit strategies reduce the risk of losing the entire investment.

Not using any exit threshold worked out remarkably well while testing the strategy, until a total loss occurred at one point. Thus, we thought about ways to handle the risk without having to state any exit strategies. For this reason we allowed to check "no-exit" strategies on just a fraction of the available investment. This means that the strategy is never closed before expiration, yet we choose the number of traded contracts $N$ such that the maximum loss is a fraction of $x \%$ of the available investment amount.

This approach led to very positive results, especially where the short strike was chosen in a very aggressive way, e.g. exactly at the money or even slightly above.

\section{Two-month strategy with shifted legs}

One special strategy implemented in our software is the so called 2M Strategy (2-month strategy), where options with two months until expiration are traded. The available investment is, however, split into two halves, and one month after opening the first leg, we are using the second half of the capital to start another leg with expiration in two months. The idea is to be able to react to market developments when investing the next half of the capital.

\section{Upper and lower bound for short strike}

It is also possible to define upper and lower bounds for the short strike. If the short strike calculation would result in a value outside this interval, then the respective boundary is used instead.

\section{Re-testing successful strategies}

In the section "overview and discussion of the test results", the previous paper (Larcher, Del Chicca, and Szölgyenyi, 2013) included a listing of the most successful strategies in terms of return per annum as well as in terms of Sharpe ratio. Building on this knowledge we were strongly interested in finding out how these strategies fared in the period reviewed for the present paper, that is, from January 2010 to August 2020. The results should help to understand how stable such parameter choices are in terms of profitability. From the previous results we know that:

- $\quad$ strategies based on

- a short strike very close to the current S\&P500's value (e.g. $\left.K_{1}=S_{0}, K_{1}=(1-0.1 \cdot V I X) \cdot S_{0}\right)$

- an exit strategy, which stops the current trades somewhere between 5\% to $20 \%$ loss, and

- a long strike of $0.97 \cdot K_{1}$ or $0.95 \cdot K_{1}$ 
performed best in terms of return p.a.

- $\quad$ strategies based on

- either the "minimum premium" approach, or

- current volatility and further out-the-money

(e.g. $K_{1}=(1-0.7 \cdot V I X) \cdot S_{0}$ or $\left.K_{-} 1=(1-0.5 \cdot h v) \cdot S_{-} 0\right)$

- a long strike of $0.91 \cdot K_{1}$ or $0.93 \cdot K_{1}$ and

- an exit strategy somewhere between 5\% and 30\% loss

performed best in terms of Sharpe ratio

For all the following re-tests we fix the standard parameters as follows:

- initial investment capital: 100,000 USD

- start of period: January 2010

- end of period: August 2020

- standard 1-month strategy, i.e. from each month's third Friday to the subsequent month's third Friday

- transaction costs: 5 USD per contract

\section{Re-testing "best strategies in terms of return p.a."}

To re-test the best strategies in terms of return we ran the strategy on arbitrary combinations of the parameters in Table 3. Figure 10 shows a return-volatility diagram of these combinations. The average return p.a. of these strategies was $7.61 \%$, the average Sharpe ratio was 0.1191 . As can be seen, these choices of strategy parameters are rather volatile (as is to be expected), with high potential for both profits and losses. 
A. Brunhuemer, G. Larcher, L. Larcher / ACRN Journal of Finance and Risk Perspectives 10 (2021) Special Issue $18^{\text {th }}$ FRAP Conference, 166-203

Table 3. Parameter combinations for re-testing of best strategies in terms of return p.a.

\begin{tabular}{llc}
\hline Short strike $\boldsymbol{K}_{\mathbf{1}}$ & Long strike $\boldsymbol{K}_{\mathbf{2}}$ & Exit \\
\hline $1.01 * \mathrm{~S} 0$ & $0.97 * \mathrm{~K} \_1$ & $5 \%$ \\
S0 & $0.95 * \mathrm{~K} \_1$ & $10 \%$ \\
& & $15 \%$ \\
& & \\
$0.98 * \mathrm{~S} 0$ & & \\
$(1-0.1 * \mathrm{vix}) * \mathrm{~S} 0$ & & \\
$(1-0.2 * \mathrm{vix}) * \mathrm{~S} 0$ & & \\
$(1-0.1 * \mathrm{hv}) * \mathrm{~S} 0$ & \\
$(1-0.2 * \mathrm{hv}) * \mathrm{~S} 0$ & \\
\hline
\end{tabular}

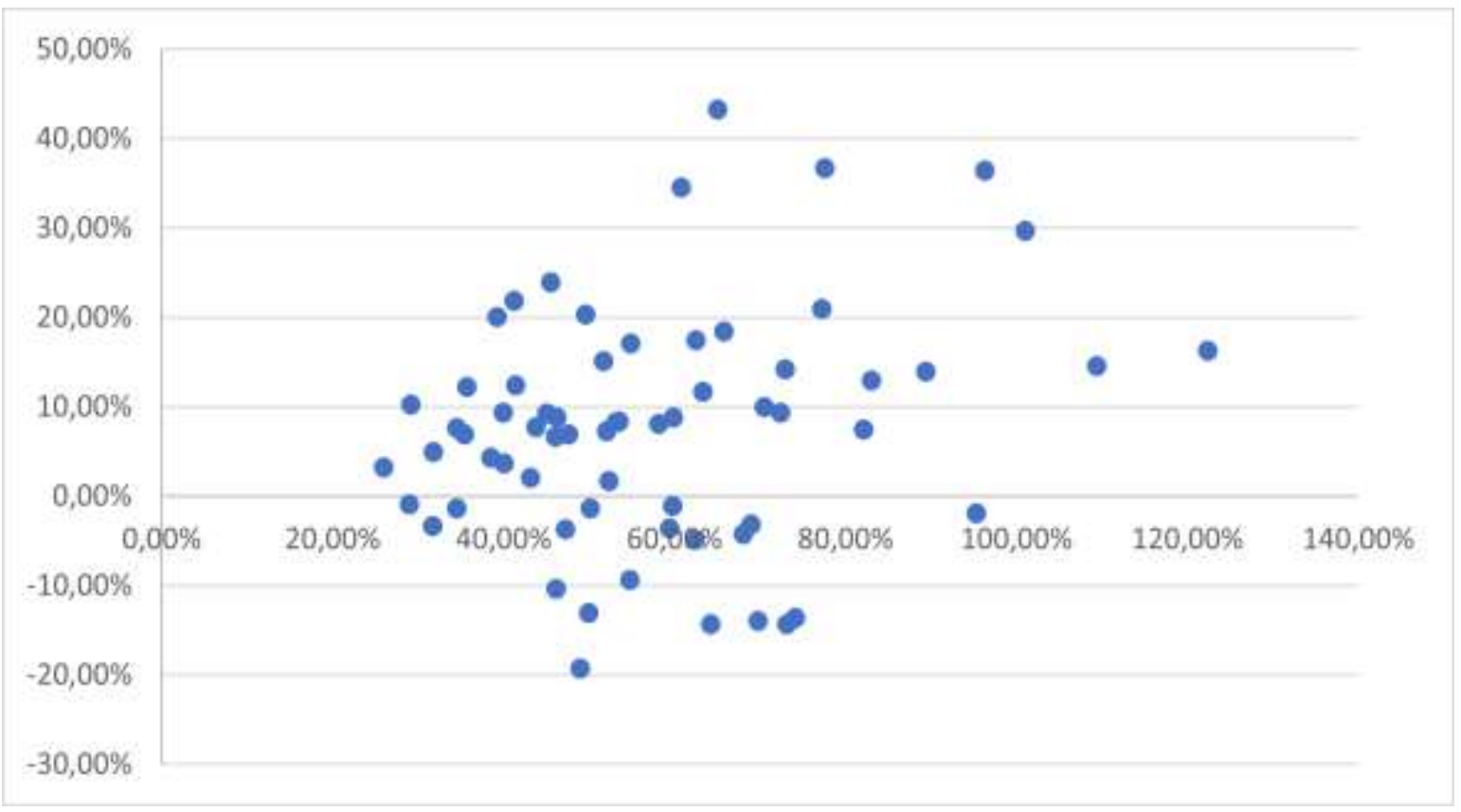

Figure 10. Return-volatility diagram showing the re-testing results of the best strategies in terms of return p.a.

Some examples of parameter choices which were in the top 10 in the previous paper's time span until 2010 and worked well in the currently considered time span are provided in Table 4. The results for these specific choices are obviously worse than in the early 2000s, which is not surprising since these were the top results of the previous backtests. 
Table 4. Examples of parameter combination which worked well until 2010 and 2020 in terms of return p.a

\begin{tabular}{|c|c|c|c|c|c|}
\hline Short strike $K_{1}$ & Long strike $K_{2}$ & Exit & Return (p.a.) & Sharpe & Prev. return (p.a.) \\
\hline $0.98 \cdot S_{0}$ & $0.95 \cdot K_{1}$ & $20 \%$ & $34.50 \%$ & 0.5351 & $62.30 \%(2000-2009)$ \\
\hline $\begin{array}{c}(1-0.1 \cdot V I X) \\
S_{0}\end{array}$ & $0.95 \cdot K_{1}$ & $15 \%$ & $17.05 \%$ & 0.2742 & $59.95 \%(2000-2009)$ \\
\hline$S_{0}$ & $0.95 \cdot K_{1}$ & $5 \%$ & $7.70 \%$ & 0.1053 & $61.90 \%(2000-2009)$ \\
\hline
\end{tabular}

\section{Re-testing "best strategies in terms of Sharpe ratio"}

In our re-tests of the best strategies in terms of Sharpe ratio we used the parameter combinations shown in Table 5. The results over all of these combinations are illustrated in a return-volatility diagram in Figure 11. The results showed much less volatile returns, which in turn also leads to a higher Sharpe ratio. The average return p.a. is $6.86 \%$ with an average Sharpe ratio of 0.3661 . What is striking, however, is that especially the minimum-premium approach consistently yields much more stable results. To illustrate this we added Figure 12, where only the minimum-premium strategies are plotted. Note that the average return of $8.28 \%$ and the average Sharpe ratio of 0.434 are also better in these types of strategies.

Table 5. Parameter combinations for re-testing of best strategies in terms of Sharpe ratio

\begin{tabular}{llc}
\hline Short-Strike & Long-Strike & Exit \\
\hline minimum premium $1-6 \%$ & $0.91 * \mathrm{~K} \_1$ & $5 \%$ \\
$(1-0.1 * \mathrm{hv}) * \mathrm{~S} 0$ & $0.93 * \mathrm{~K} \_1$ & $10 \%$ \\
$(1-0.2 * \mathrm{hv}) * \mathrm{~S} 0$ & & $15 \%$ \\
& & $20 \%$ \\
\hline$(1-0.8 * \mathrm{vix}) * \mathrm{~S} 0$ & $0.97 * \mathrm{~K} \_1$ & $20 \%$ \\
$(1-0.6 * \mathrm{vix}) * \mathrm{~S} 0$ & & $25 \%$ \\
$(1-0.7 * \mathrm{vix}) * \mathrm{~S} 0$ & & $30 \%$ \\
\hline
\end{tabular}




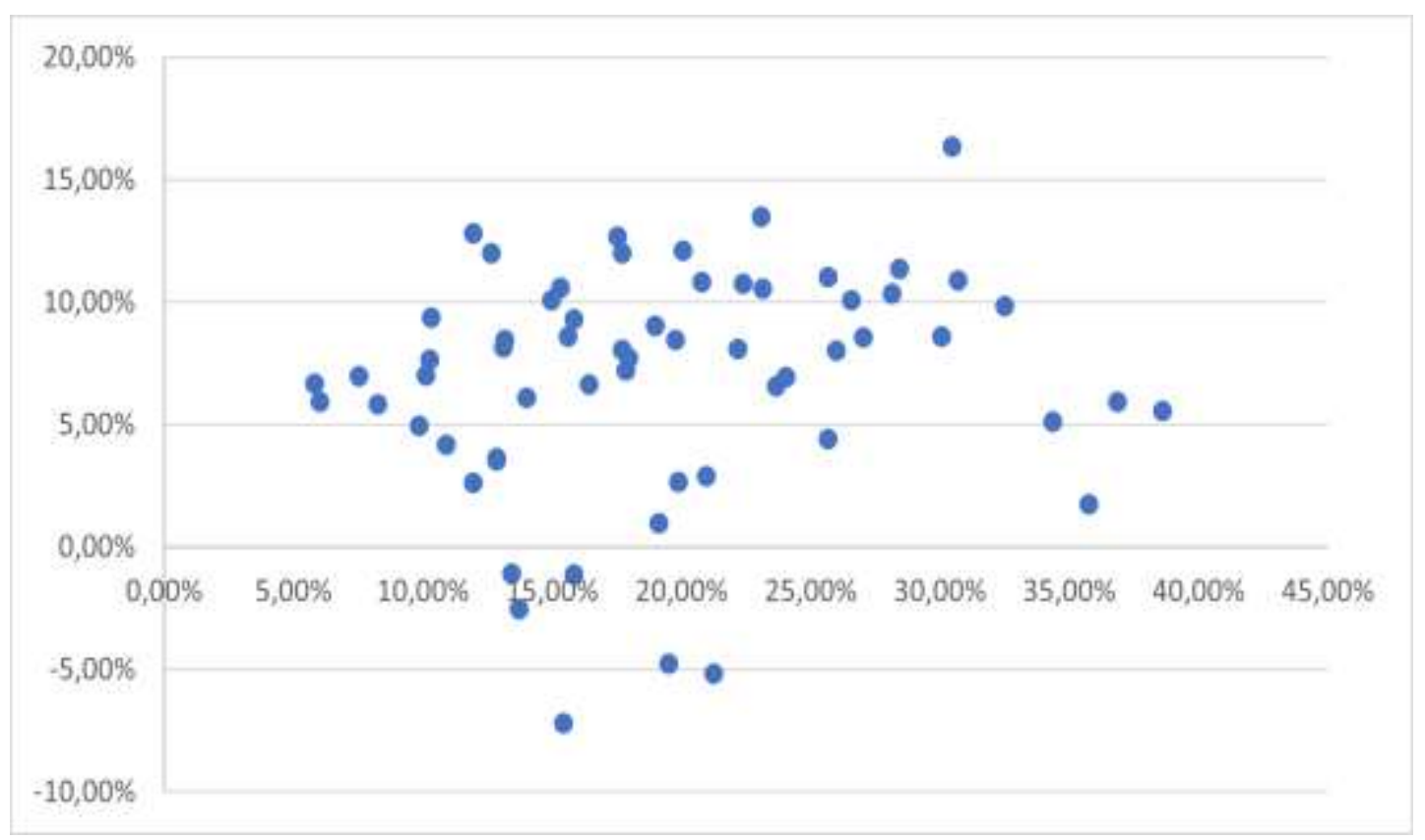

Figure 11. Return-volatility diagram showing the re-testing results of the best strategies in terms of Sharpe ratio

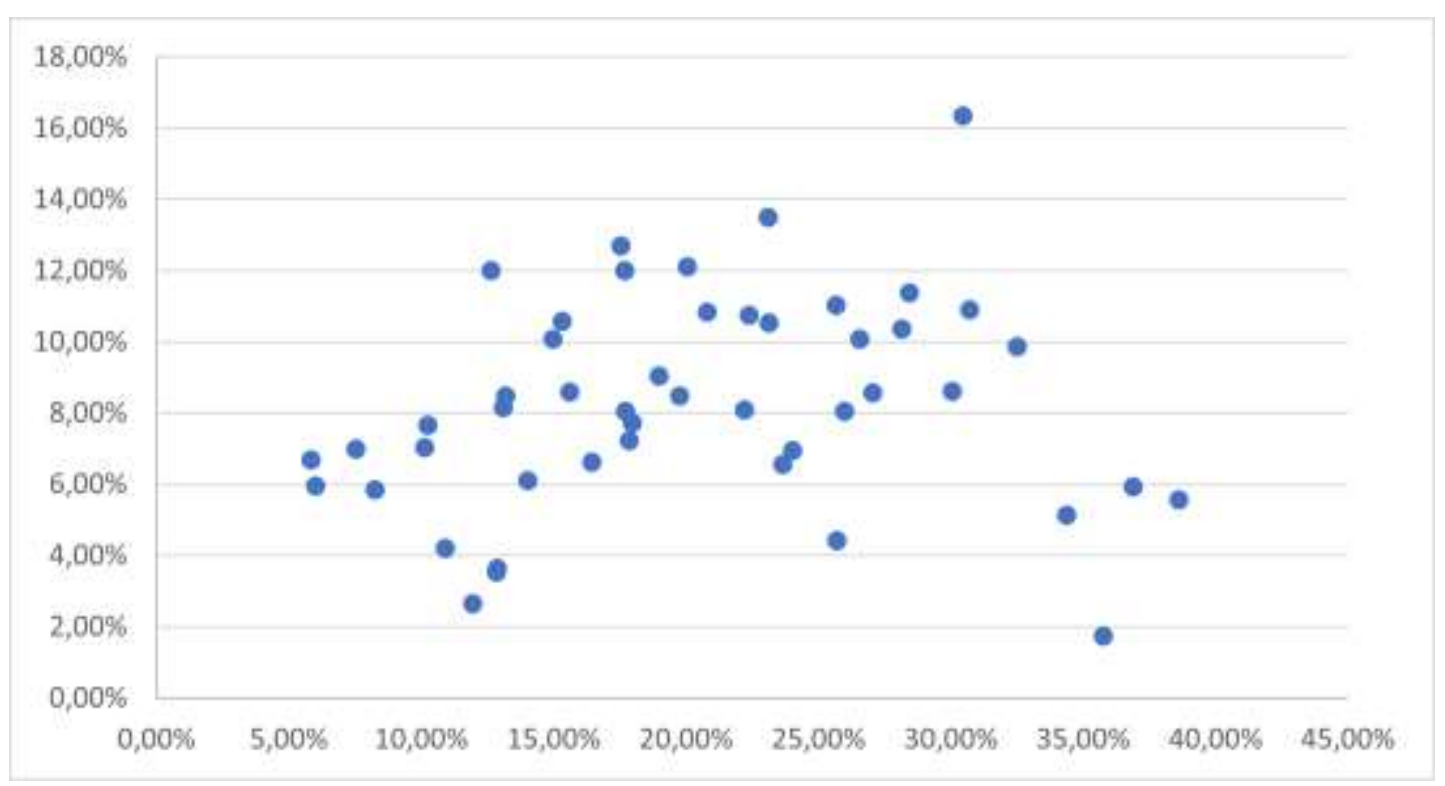

Figure 12. Return-volatility diagram showing the re-testing results of the minimum-premium strategies

When re-testing the best strategies in terms of return, we also looked at some specific parameter choices in terms of Sharpe ratio that worked well in both considered time spans before 2010 and afterwards. These are presented in Table 6. Again the results deteriorated in the later period but were still clearly positive. 
Table 6. Examples of parameter combination which worked well until 2010 as well as between 2010 and 2020 in terms of Sharpe ratio

\begin{tabular}{ccc|cc|c}
\hline Short strike & Long strike $K_{2}$ & Exit & Return (p.a.) & Sharpe & Prev. return (p.a.) \\
\hline MP 3\% & $0.91 \cdot K_{1}$ & $5 \%$ & $11.99 \%$ & 0.7903 & $31.40 \%(1990-2009)$ \\
MP 5\% & $0.91 \cdot K_{1}$ & $5 \%$ & $12.00 \%$ & 0.5651 & $43.15 \%(1990-2009)$ \\
MP 4\% & $0.91 \cdot K_{1}$ & $5 \%$ & $10.58 \%$ & 0.5602 & $33.13 \%(2000-2009)$ \\
\hline
\end{tabular}

\section{Overview of results in the last decade}

In the following we are going to provide an overview of the results of our extensive testing of various combinations of parameter choices. For all of the following tests we used these fixed parameter settings:

- initial investment capital: 100,000 USD

- start of period: January 15, 2010

- latest possible period start: July 27, 2020

- transaction costs: 5 USD per contract

Additionally we combine possible choices from among these variable parameters:

-period length: 3 days, 7 days, 14 days, 30 days

- short strikes

- minimum premium: $1 \%, 2 \%, 3 \%, 4 \%, 5 \%, 6 \%$

- values $x \cdot S_{0}$ with $x \in\{0.88,0.9,0.92,0.94,0.96,0.98,1,1.02,1.04\}$

- values $(1-x \cdot V I X) S_{0}$ with $x \in\{0.1,0.2,0.3,0.4,0.5,0.6,0.7,0.8\}$

- values $(1-x \cdot h v) S_{0}$ with $x \in\{0.1,0.2,0.3,0.4,0.5,0.6,0.7,0.8\}$

•long strikes with values $x \cdot K_{1}$ with $x \in\{0.9,0.92,0.94,0.96,0.98\}$

•exit thresholds at 5\%,10\%,15\%,20\%,25\%,30\%

All results can be found on the website at: https://app.lsqf.org/option-strategies/short-strategy-results

An overview of the best results in terms of the Sharpe ratio on the one hand, and in terms of return per annum on the other hand, is presented in Tables 7 and 8 .

\section{Best strategies in terms of Sharpe ratio}

Our goal was to identify groups of parameter settings which work structurally well. It was not to find specific parameter settings that worked well in exceptional cases.

- Strategies based on the minimum-premium approach combined with an early exit strategy (at 5\% or 10\% loss) and long strikes not too close at the money (between $0.9 \cdot K_{1}$ and $0.94 \cdot K_{1}$ ) worked especially well and were less volatile. Higher thresholds for the exit strategy and longer periods reduce the profit. 
- Another successful approach consists in choosing a risky short strike of $1.02 \cdot S_{0}$ or $S_{0}$ paired with an exit strategy and a long strike not too close to the money, i.e. between $0.9 \cdot K_{1}$ and $0.94 \cdot K_{1}$. What is noticeable here is that shorter periods can lead to losses.

- When choosing shorter periods the results suggest a more conservative out-of-the-money strike choice of $0.98 \cdot S_{0}$, again combined with an exit strategy and long strikes as before.

Table 7 lists the top 50 strategies in terms of Sharpe ratio.

\section{Best strategies in terms of return per annum}

When looking at the best strategies in terms of return per annum (see excerpt in Table 8) there are a few things that stand out:

- Short strike choices are on the very risky side, especially where they are at the money, but in most cases even above that level.

- Longer periods - i.e. 14 or 30 days - are more common in this table than in the "best strategies in terms of Sharpe ratio". This is probably due to the riskier (higher) short strike choices and the need for the underlying asset to increase until expiration.

- In general, and as is to be expected, the maximum drawdown is much higher than for the "best strategies in terms of Sharpe ratio".

What was astonishing for us is the poor performance of strategies based on 3-day periods. Not only are those strategies missing in the top lists (Tables 7 and 8), but their results were also mostly negative. However, we want to emphasize that checking an option's values on a daily basis might be too rough a subdivision to work reliably for such short-term observations.

\section{Scenario testing}

Finally, we take a closer look at some specific periods between January 2010 and August 2020 in which the S\&P500 exhibited different characteristics.

- S1 (2010-2017): The performance of the S\&P500 in these 8 years is best described as steadily growing, without much volatility. Even though losses did not occur often, the options' prices were rather low, which is not positive for the strategy per se.

- S2 (2018): This year consisted of big and sudden losses in the S\&P500's value at the start and the end of the year, and a steady growth in between. In terms of the put-write strategy, many sudden losses, such as the ones that occurred in this period, are the worst possible scenario.

- S3 (January 2020 - June 2020): Early 2020 was a really special year, with a huge loss for the S\&P500 in March and a fast recovery by June, amid very high volatility in the markets. Option prices were enormously high in this period.

Since these three periods are so fundamentally different, our interest now lies in finding strategies which worked well in each of these periods. We aim to find tendencies as to which strategies work well in a wide range of scenarios. The minimum-premium approaches seem to have very balanced outcomes throughout the different scenarios, while 
the other variants mostly have one scenario that leads to clearly negative results. Examples of such strategies are shown in Table 9.

Table 6. Strategies which worked relatively well in periods of different characteristics

\begin{tabular}{cccc|ccc}
\hline Short strike & Long strike & Exit & Period & S1 Return (p.a.) & S2 return (p.a.) & S3 return (p.a.) \\
\hline MP 3\% & $0.92 * \mathrm{k}$ & $5.00 \%$ & $7 \mathrm{~d}$ & $52.81 \%$ & $29.92 \%$ & $34.45 \%$ \\
MP 5\% & $0.92 * \mathrm{k}$ & $5.00 \%$ & $7 \mathrm{~d}$ & $66.55 \%$ & $-0,76 \%$ & $164.68 \%$ \\
$(1-0.3 * \mathrm{v}) * \mathrm{~s}$ & $0.92 * \mathrm{k}$ & $5.00 \%$ & $30 \mathrm{~d}$ & $28.89 \%$ & $11.89 \%$ & $-12.02 \%$ \\
$1.02 * \mathrm{~s}$ & $0.9 \mathrm{k}$ & $5.00 \%$ & $14 \mathrm{~d}$ & $114.56 \%$ & $-9.81 \%$ & $4488.55 \%$ \\
$0.94 * \mathrm{~s}$ & $0.9 * \mathrm{k}$ & $10.00 \%$ & $30 \mathrm{~d}$ & $36.74 \%$ & $12.09 \%$ & $-14.56 \%$ \\
\hline
\end{tabular}

Especially interesting is the first strategy, not only since it is the best strategy overall in terms of Sharpe ratio, but also since it worked well in all of the three different periods. Because of this remarkable performance we provide some additional insights into this specific strategy and its performance in Figure 13. For better visibility we also added the performance of that strategy on a logarithmic scale in Figure 14. Between 2010 and August 2020 this strategy had 324 periods with positive results and 109 periods with negative results. For more in-depth details we refer to our analysis software on app.lsqf.org.

The second notable result is the S3 return of the "1.02.s" strategy in Table 9. At first glance the $4488.55 \%$ return (p.a.) might seem like an error, however, the very high volatility in this specific time span led to this enormously high profit (486.80\% from January to June). Calculating the per-annum return thus gives us $4488.55 \%$.

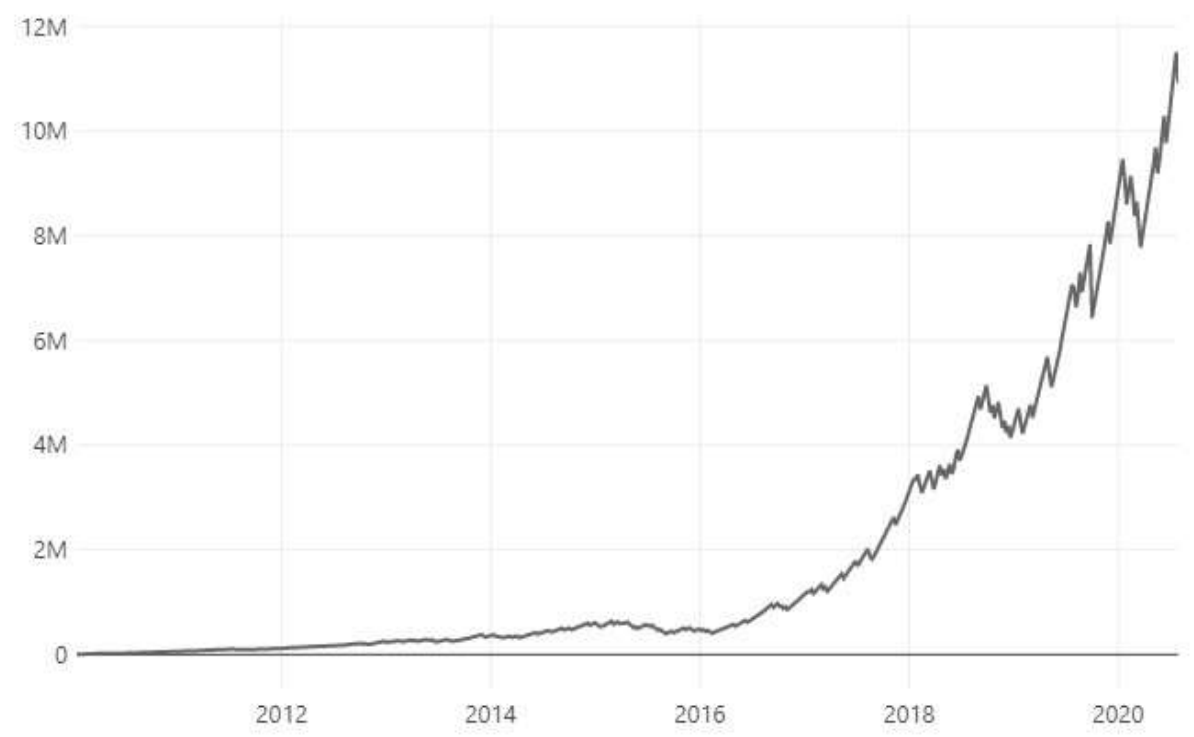

Figure 13. Profit of the $3 \%$ MP strategy with $0.92 \cdot K_{1}$, exit at $5 \%$ and 7 d period from 2010 to August 2020 (lin. scale) 
A. Brunhuemer, G. Larcher, L. Larcher / ACRN Journal of Finance and Risk Perspectives 10 (2021) Special Issue $18^{\text {th }}$ FRAP Conference, 166-203

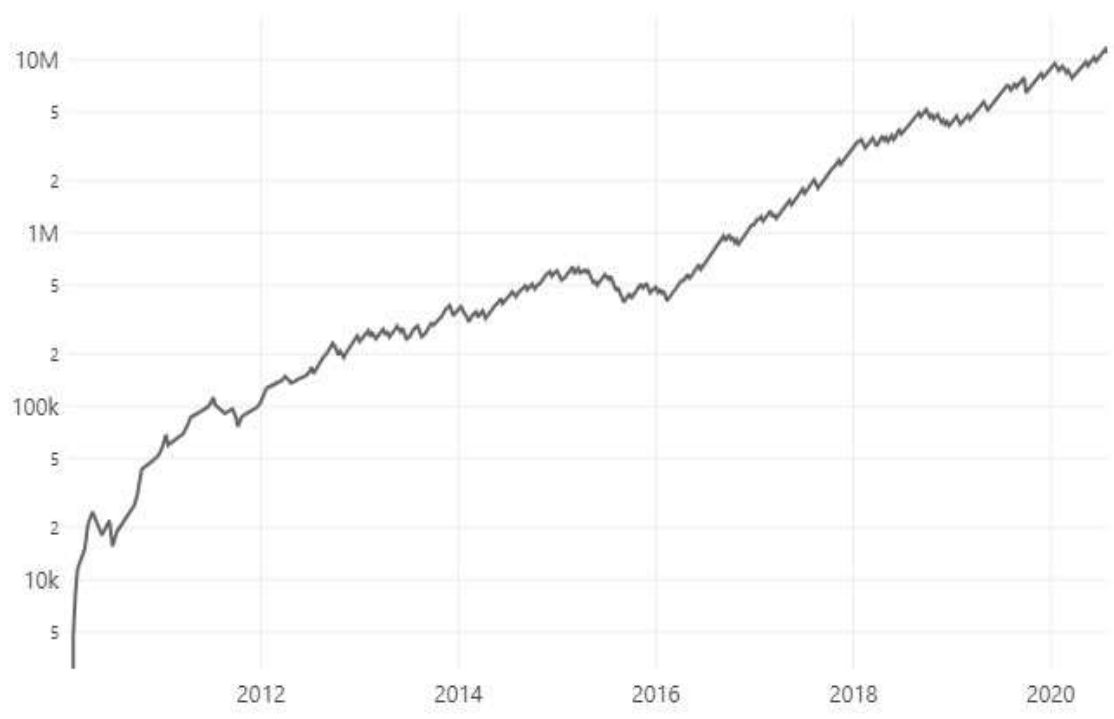

Figure 14. Profit of the $3 \%$ MP strategy with $0.92 \cdot K_{1}$, exit at $5 \%$ and 7 d period from 2010 to August 2020 (log scale) 
Table 7. Best strategies between January 2010 and August 2020 in terms of Sharpe ratio

\begin{tabular}{|c|c|c|c|c|c|c|c|}
\hline Short strike & Long strike & Exit & Period & Return p.a. & Max drawdown & Volatility p.a. & Sharpe \\
\hline MP $3.00 \%$ & $0.92 * \mathrm{k}$ & $5.00 \%$ & $7 \mathrm{~d}$ & $56.24 \%$ & $-32.64 \%$ & $24.23 \%$ & 2.2383 \\
\hline MP $5.00 \%$ & $0.92 * \mathrm{k}$ & $5.00 \%$ & $7 \mathrm{~d}$ & $67.76 \%$ & $-42.41 \%$ & $32.10 \%$ & 2.0483 \\
\hline MP $4.00 \%$ & $0.92^{*} \mathrm{k}$ & $5.00 \%$ & $7 \mathrm{~d}$ & $59.81 \%$ & $-44.86 \%$ & $28.76 \%$ & 2.0102 \\
\hline MP $2.00 \%$ & $0.9^{*} \mathrm{k}$ & $5.00 \%$ & $7 \mathrm{~d}$ & $37.80 \%$ & $-34.92 \%$ & $18.89 \%$ & 1.8950 \\
\hline MP $3.00 \%$ & $0.9^{*} \mathrm{k}$ & $5.00 \%$ & $7 \mathrm{~d}$ & $46.76 \%$ & $-26.33 \%$ & $24.15 \%$ & 1.8531 \\
\hline MP $2.00 \%$ & $0.92 * k$ & $5.00 \%$ & $7 \mathrm{~d}$ & $37.00 \%$ & $-21.04 \%$ & $19.10 \%$ & 1.8329 \\
\hline MP $5.00 \%$ & $0.94^{*} \mathrm{k}$ & $5.00 \%$ & $7 \mathrm{~d}$ & $61.80 \%$ & $-42.48 \%$ & $33.22 \%$ & 1.8000 \\
\hline $1.02{ }^{*} \mathrm{~s}$ & $0.9^{*} \mathrm{k}$ & $5.00 \%$ & $14 \mathrm{~d}$ & $131.11 \%$ & $-74.24 \%$ & $73.35 \%$ & 1.7601 \\
\hline$\left(1-0.7^{*} \mathrm{v}\right)^{*} \mathrm{~s}$ & $0.9 * \mathrm{k}$ & $30.00 \%$ & $14 \mathrm{~d}$ & $4.12 \%$ & $-0.96 \%$ & $1.23 \%$ & 1.7215 \\
\hline MP $6.00 \%$ & $0.96^{*} \mathrm{k}$ & $5.00 \%$ & $7 \mathrm{~d}$ & $67.79 \%$ & $-39.26 \%$ & $38.38 \%$ & 1.7141 \\
\hline MP $4.00 \%$ & $0.94^{*} \mathrm{k}$ & $5.00 \%$ & $7 \mathrm{~d}$ & $51.72 \%$ & $-46.75 \%$ & $29.59 \%$ & 1.6805 \\
\hline s & $0.92^{*} \mathrm{k}$ & $5.00 \%$ & $7 \mathrm{~d}$ & $86.39 \%$ & $-47.58 \%$ & $50.22 \%$ & 1.6804 \\
\hline MP $3.00 \%$ & $0.96^{*} \mathrm{k}$ & $5.00 \%$ & $7 \mathrm{~d}$ & $43.81 \%$ & $-35.00 \%$ & $24.96 \%$ & 1.6751 \\
\hline s & $0.9^{*} \mathrm{k}$ & $5.00 \%$ & $7 \mathrm{~d}$ & $74.84 \%$ & $-40.11 \%$ & $43.70 \%$ & 1.6670 \\
\hline $1.02 * \mathrm{~s}$ & $0.92^{*} \mathrm{k}$ & $25.00 \%$ & $30 \mathrm{~d}$ & $159.49 \%$ & $-72.78 \%$ & $95.97 \%$ & 1.6410 \\
\hline $1.02 *_{\mathrm{s}}$ & $0.9^{*} \mathrm{k}$ & $10.00 \%$ & $14 \mathrm{~d}$ & $140.77 \%$ & $-75.65 \%$ & $84.67 \%$ & 1.6390 \\
\hline MP $5.00 \%$ & $0.9^{*} \mathrm{k}$ & $5.00 \%$ & $7 \mathrm{~d}$ & $53.24 \%$ & $-42.65 \%$ & $31.85 \%$ & 1.6088 \\
\hline s & $0.96^{*} \mathrm{k}$ & $5.00 \%$ & $7 \mathrm{~d}$ & $124.42 \%$ & $-59.10 \%$ & $77.76 \%$ & 1.5744 \\
\hline $1.02 * \mathrm{~s}$ & $0.92^{*} \mathrm{k}$ & $5.00 \%$ & $14 \mathrm{~d}$ & $143.92 \%$ & $-88.20 \%$ & $90.27 \%$ & 1.5721 \\
\hline MP $4.00 \%$ & $0.9^{*} \mathrm{k}$ & $5.00 \%$ & $7 \mathrm{~d}$ & $47.26 \%$ & $-42.08 \%$ & $28.93 \%$ & 1.5642 \\
\hline MP $3.00 \%$ & $0.94^{*} \mathrm{k}$ & $5.00 \%$ & $7 \mathrm{~d}$ & $41.18 \%$ & $-39.15 \%$ & $25.40 \%$ & 1.5428 \\
\hline MP $5.00 \%$ & $0.96^{*} \mathrm{k}$ & $5.00 \%$ & $7 \mathrm{~d}$ & $54.71 \%$ & $-40.69 \%$ & $34.29 \%$ & 1.5371 \\
\hline s & $0.9 * \mathrm{k}$ & $5.00 \%$ & $14 \mathrm{~d}$ & $65.31 \%$ & $-44.78 \%$ & $41.25 \%$ & 1.5347 \\
\hline$\left(1-0.3^{*} \mathrm{v}\right)^{*} \mathrm{~s}$ & $0.9^{*} \mathrm{k}$ & $5.00 \%$ & $14 \mathrm{~d}$ & $20.22 \%$ & $-15.25 \%$ & $11.92 \%$ & 1.5280 \\
\hline $1.02{ }^{*} \mathrm{~s}$ & $0.9^{*} \mathrm{k}$ & $5.00 \%$ & $30 \mathrm{~d}$ & $88.32 \%$ & $-45.92 \%$ & $56.66 \%$ & 1.5235 \\
\hline $\mathrm{s}$ & $0.94^{*} \mathrm{k}$ & $5.00 \%$ & $7 \mathrm{~d}$ & $89.76 \%$ & $-57.32 \%$ & $58.10 \%$ & 1.5105 \\
\hline MP $6.00 \%$ & $0.96^{*} \mathrm{k}$ & $10.00 \%$ & $7 \mathrm{~d}$ & $78.32 \%$ & $-52.60 \%$ & $51.02 \%$ & 1.4961 \\
\hline$\left(1-0.2^{*} \mathrm{v}\right)^{*} \mathrm{~s}$ & $0.9^{*} \mathrm{k}$ & $5.00 \%$ & $30 \mathrm{~d}$ & $33.18 \%$ & $-23.06 \%$ & $20.99 \%$ & 1.4856 \\
\hline MP $6.00 \%$ & $0.92 * \mathrm{k}$ & $5.00 \%$ & $7 \mathrm{~d}$ & $55.46 \%$ & $-35.93 \%$ & $36.01 \%$ & 1.4845 \\
\hline MP $4.00 \%$ & $0.96^{*} \mathrm{k}$ & $5.00 \%$ & $7 \mathrm{~d}$ & $45.74 \%$ & $-44.93 \%$ & $29.80 \%$ & 1.4677 \\
\hline$(1-0.2 * h) *_{\mathrm{s}}$ & $0.9^{*} \mathrm{k}$ & $10.00 \%$ & $30 d$ & $44.66 \%$ & $-24.31 \%$ & $29.13 \%$ & 1.4647 \\
\hline$\left(1-0.2^{*} v\right)^{*} \mathrm{~s}$ & $0.9^{*} \mathrm{k}$ & $10.00 \%$ & $30 d$ & $38.94 \%$ & $-31.01 \%$ & $25.28 \%$ & 1.4611 \\
\hline $1.02 * \mathrm{~s}$ & $0.92^{*} \mathrm{k}$ & $10.00 \%$ & $30 d$ & $114.56 \%$ & $-54.19 \%$ & $77.11 \%$ & 1.4598 \\
\hline $1.02 * \mathrm{~s}$ & $0.92 * \mathrm{k}$ & $10.00 \%$ & $14 \mathrm{~d}$ & $151.17 \%$ & $-88.84 \%$ & $102.25 \%$ & 1.4589 \\
\hline$\left(1-0.2^{*} \mathrm{v}\right)^{*} \mathrm{~s}$ & $0.9 * \mathrm{k}$ & $10.00 \%$ & $14 \mathrm{~d}$ & $35.36 \%$ & $-35.32 \%$ & $23.01 \%$ & 1.4499 \\
\hline MP $4.00 \%$ & $0.94^{*} \mathrm{k}$ & $10.00 \%$ & $7 \mathrm{~d}$ & $59.23 \%$ & $-55.32 \%$ & $39.50 \%$ & 1.4487 \\
\hline$\left(1-0.3^{*} v\right)^{*} \mathrm{~s}$ & $0.92^{*} \mathrm{k}$ & $5.00 \%$ & $30 d$ & $26.57 \%$ & $-19.63 \%$ & $17.00 \%$ & 1.4457 \\
\hline $1.02{ }^{\mathrm{s}}$ & $0.9^{*} \mathrm{k}$ & $25.00 \%$ & $30 \mathrm{~d}$ & $118.45 \%$ & $-74.44 \%$ & $81.61 \%$ & 1.4269 \\
\hline MP $4.00 \%$ & $0.96^{*} \mathrm{k}$ & $10.00 \%$ & $7 \mathrm{~d}$ & $58.37 \%$ & $-58.98 \%$ & $39.81 \%$ & 1.4158 \\
\hline MP $5.00 \%$ & $0.94^{*} \mathrm{k}$ & $10.00 \%$ & $7 \mathrm{~d}$ & $64.43 \%$ & $-64.66 \%$ & $44.46 \%$ & 1.4040 \\
\hline$(1-0.1 * v)^{*} \mathrm{~s}$ & $0.94^{*} \mathrm{k}$ & $5.00 \%$ & $7 \mathrm{~d}$ & $47.74 \%$ & $-38.36 \%$ & $32.58 \%$ & 1.4040 \\
\hline s & $0.92^{*} \mathrm{k}$ & $10.00 \%$ & $7 \mathrm{~d}$ & $90.87 \%$ & $-50.10 \%$ & $63.78 \%$ & 1.3935 \\
\hline$(1-0.1 * v)^{*} \mathrm{~s}$ & $0.9^{*} \mathrm{k}$ & $20.00 \%$ & $30 d$ & $59.77 \%$ & $-36.18 \%$ & $41.70 \%$ & 1.3852 \\
\hline$\left(1-0.3^{*} h\right)^{*} \mathrm{~s}$ & $0.92 * \mathrm{k}$ & $15.00 \%$ & $30 d$ & $42.53 \%$ & $-33.25 \%$ & $29.30 \%$ & 1.3832 \\
\hline s & $0.9 * \mathrm{k}$ & $10.00 \%$ & $30 \mathrm{~d}$ & $67.43 \%$ & $-47.27 \%$ & $47.36 \%$ & 1.3815 \\
\hline MP $6.00 \%$ & $0.9^{*} \mathrm{k}$ & $5.00 \%$ & $7 \mathrm{~d}$ & $49.32 \%$ & $-40.63 \%$ & $34.48 \%$ & 1.3725 \\
\hline $0.94 * s$ & $0.9^{*} \mathrm{k}$ & $10.00 \%$ & $30 d$ & $30.44 \%$ & $-19.13 \%$ & $20.86 \%$ & 1.3633 \\
\hline MP $6.00 \%$ & $0.94^{*} \mathrm{k}$ & $5.00 \%$ & $7 \mathrm{~d}$ & $52.12 \%$ & $-37.88 \%$ & $36.82 \%$ & 1.3613 \\
\hline $1.02 *_{\mathrm{s}}$ & $0.9^{*} \mathrm{k}$ & $20.00 \%$ & $30 d$ & $106.90 \%$ & $-81.19 \%$ & $77.40 \%$ & 1.3553 \\
\hline$(1-0.1 * v) * \mathrm{~s}$ & $0.92^{*} \mathrm{k}$ & $25.00 \%$ & $30 \mathrm{~d}$ & $68.85 \%$ & $-43.89 \%$ & $49.55 \%$ & 1.3491 \\
\hline
\end{tabular}


Table 8. Best strategies between January 2010 and August 2020 in terms of return per anno

\begin{tabular}{|c|c|c|c|c|c|c|c|}
\hline Short strike & Long strike & Exit & Period & return p.a. & Max drawdown & Volatility p.a. & Sharpe \\
\hline $1.02 * \mathrm{~s}$ & $0.92^{*} \mathrm{k}$ & $25.00 \%$ & $30 \mathrm{~d}$ & $159.49 \%$ & $-72.78 \%$ & $95.97 \%$ & 1.6410 \\
\hline $1.02 * \mathrm{~s}$ & $0.92 * \mathrm{k}$ & $10.00 \%$ & $14 \mathrm{~d}$ & $151.17 \%$ & $-88.84 \%$ & $102.25 \%$ & 1.4589 \\
\hline $1.02 * \mathrm{~s}$ & $0.92 * k$ & $5.00 \%$ & $14 \mathrm{~d}$ & $143.92 \%$ & $-88.20 \%$ & $90.27 \%$ & 1.5721 \\
\hline $1.02 * \mathrm{~s}$ & $0.9^{*} \mathrm{k}$ & $10.00 \%$ & $14 \mathrm{~d}$ & $140.77 \%$ & $-75.65 \%$ & $84.67 \%$ & 1.6390 \\
\hline $1.04 * \mathrm{~s}$ & $0.9^{*} \mathrm{k}$ & $15.00 \%$ & $14 \mathrm{~d}$ & $139.22 \%$ & $-94.65 \%$ & $133.33 \%$ & 1.0291 \\
\hline $1.02 * \mathrm{~s}$ & $0.94 * k$ & $25.00 \%$ & $30 \mathrm{~d}$ & $136.18 \%$ & $-83.43 \%$ & $116.82 \%$ & 1.1486 \\
\hline $1.02 *_{\mathrm{s}}$ & $0.94 * \mathrm{k}$ & $20.00 \%$ & $30 d$ & $134.02 \%$ & $-83.43 \%$ & $112.03 \%$ & 1.1784 \\
\hline $1.02 *_{s}$ & $0.9^{*} \mathrm{k}$ & $5.00 \%$ & $14 \mathrm{~d}$ & $131.11 \%$ & $-74.24 \%$ & $73.35 \%$ & 1.7601 \\
\hline s & $0.98 * \mathrm{k}$ & $5.00 \%$ & $7 \mathrm{~d}$ & $127.84 \%$ & $-71.09 \%$ & $106.17 \%$ & 1.1852 \\
\hline s & $0.96 * \mathrm{k}$ & $5.00 \%$ & $7 \mathrm{~d}$ & $124.42 \%$ & $-59.10 \%$ & $77.76 \%$ & 1.5744 \\
\hline $1.02{ }^{*} \mathrm{~s}$ & $0.94^{*} \mathrm{k}$ & $30.00 \%$ & $30 \mathrm{~d}$ & $122.36 \%$ & $-85.46 \%$ & $122.13 \%$ & 0.9855 \\
\hline $1.04 * \mathrm{~s}$ & $0.9^{*} \mathrm{k}$ & $10.00 \%$ & $14 \mathrm{~d}$ & $122.26 \%$ & $-95.43 \%$ & $125.14 \%$ & 0.9610 \\
\hline $\mathrm{s}$ & $0.94 * \mathrm{k}$ & $25.00 \%$ & $14 \mathrm{~d}$ & $120.80 \%$ & $-91.46 \%$ & $95.55 \%$ & 1.2433 \\
\hline $1.02{ }^{*} \mathrm{~s}$ & $0.9^{*} \mathrm{k}$ & $25.00 \%$ & $30 \mathrm{~d}$ & $118.45 \%$ & $-74.44 \%$ & $81.61 \%$ & 1.4269 \\
\hline $1.02 \mathrm{ks}$ & $0.94^{*} \mathrm{k}$ & $5.00 \%$ & $30 \mathrm{~d}$ & $115.86 \%$ & $-83.43 \%$ & $86.99 \%$ & 1.3089 \\
\hline $1.02 \% \mathrm{~s}$ & $0.9 * \mathrm{k}$ & $20.00 \%$ & $14 \mathrm{~d}$ & $115.77 \%$ & $-91.46 \%$ & $102.59 \%$ & 1.1090 \\
\hline $1.02 * \mathrm{~s}$ & $0.92^{*} \mathrm{k}$ & $10.00 \%$ & $30 \mathrm{~d}$ & $114.56 \%$ & $-54.19 \%$ & $77.11 \%$ & 1.4598 \\
\hline $1.02 \%_{\mathrm{s}}$ & $0.9^{*} \mathrm{k}$ & $30.00 \%$ & $30 d$ & $113.38 \%$ & $-79.23 \%$ & $83.32 \%$ & 1.3368 \\
\hline $1.02 \% \mathrm{~s}$ & $0.94 * k$ & $15.00 \%$ & $30 d$ & $112.88 \%$ & $-83.42 \%$ & $104.70 \%$ & 1.0590 \\
\hline $1.02 * \mathrm{~s}$ & $0.92 * \mathrm{k}$ & $30.00 \%$ & $30 \mathrm{~d}$ & $111.99 \%$ & $-83.23 \%$ & $101.73 \%$ & 1.0812 \\
\hline $1.02 *_{\mathrm{s}}$ & $0.92^{*} \mathrm{k}$ & $20.00 \%$ & $30 \mathrm{~d}$ & $111.77 \%$ & $-79.66 \%$ & $91.14 \%$ & 1.2044 \\
\hline $1.02 * \mathrm{~s}$ & $0.92 * \mathrm{k}$ & $20.00 \%$ & $14 \mathrm{~d}$ & $109.75 \%$ & $-91.18 \%$ & $119.89 \%$ & 0.8987 \\
\hline $1.02 \% \mathrm{~s}$ & $0.92^{*} \mathrm{k}$ & $15.00 \%$ & $14 \mathrm{~d}$ & $108.76 \%$ & $-90.04 \%$ & $112.51 \%$ & 0.9489 \\
\hline $1.04{ }^{*} \mathrm{~s}$ & $0.9^{*} \mathrm{k}$ & $5.00 \%$ & $14 \mathrm{~d}$ & $106.94 \%$ & $-95.29 \%$ & $117.03 \%$ & 0.8967 \\
\hline $1.02{ }^{*} \mathrm{~s}$ & $0.9^{*} \mathrm{k}$ & $20.00 \%$ & $30 \mathrm{~d}$ & $106.90 \%$ & $-81.19 \%$ & $77.40 \%$ & 1.3553 \\
\hline s & $0.96 * \mathrm{k}$ & $10.00 \%$ & $7 \mathrm{~d}$ & $104.57 \%$ & $-80.20 \%$ & $93.72 \%$ & 1.0945 \\
\hline $1.02 \% s$ & $0.92 * \mathrm{k}$ & $15.00 \%$ & $30 \mathrm{~d}$ & $103.88 \%$ & $-73.22 \%$ & $84.50 \%$ & 1.2057 \\
\hline $1.04^{*} \mathrm{~s}$ & $0.9^{*} \mathrm{k}$ & $25.00 \%$ & $14 \mathrm{~d}$ & $103.55 \%$ & $-98.01 \%$ & $144.76 \%$ & 0.7015 \\
\hline $1.02 *_{\mathrm{s}}$ & $0.9^{*} \mathrm{k}$ & $25.00 \%$ & $14 \mathrm{~d}$ & $101.93 \%$ & $-89.13 \%$ & $107.63 \%$ & 0.9285 \\
\hline s & $0.96 * \mathrm{k}$ & $20.00 \%$ & $7 \mathrm{~d}$ & $98.37 \%$ & $-87.84 \%$ & $122.88 \%$ & 0.7843 \\
\hline $1.02^{*} \mathrm{~s}$ & $0.9 * \mathrm{k}$ & $30.00 \%$ & $14 \mathrm{~d}$ & $96.84 \%$ & $-91.24 \%$ & $110.51 \%$ & 0.8582 \\
\hline s & $0.96^{*} \mathrm{k}$ & $25.00 \%$ & $7 \mathrm{~d}$ & $96.08 \%$ & $-94.50 \%$ & $131.82 \%$ & 0.7137 \\
\hline $1.04 * \mathrm{~s}$ & $0.9^{*} \mathrm{k}$ & $30.00 \%$ & $14 \mathrm{~d}$ & $95.68 \%$ & $-98.14 \%$ & $150.10 \%$ & 0.6241 \\
\hline $1.02{ }^{*} \mathrm{~s}$ & $0.94^{*} \mathrm{k}$ & $10.00 \%$ & $30 \mathrm{~d}$ & $94.87 \%$ & $-83.42 \%$ & $96.28 \%$ & 0.9646 \\
\hline $1.02 * \mathrm{~s}$ & $0.9^{*} \mathrm{k}$ & $15.00 \%$ & $30 \mathrm{~d}$ & $94.84 \%$ & $-65.85 \%$ & $72.86 \%$ & 1.2742 \\
\hline s & $0.96 * \mathrm{k}$ & $15.00 \%$ & $7 \mathrm{~d}$ & $94.30 \%$ & $-87.71 \%$ & $109.96 \%$ & 0.8394 \\
\hline $\mathrm{s}$ & $0.96 * \mathrm{k}$ & $25.00 \%$ & $14 \mathrm{~d}$ & $93.45 \%$ & $-92.55 \%$ & $116.15 \%$ & 0.7873 \\
\hline s & $0.92 * \mathrm{k}$ & $20.00 \%$ & $14 \mathrm{~d}$ & $93.40 \%$ & $-92.70 \%$ & $78.91 \%$ & 1.1583 \\
\hline s & $0.92^{*} \mathrm{k}$ & $30.00 \%$ & $14 \mathrm{~d}$ & $93.26 \%$ & $-87.61 \%$ & $85.17 \%$ & 1.0714 \\
\hline $1.02 \% \mathrm{~s}$ & $0.9^{*} \mathrm{k}$ & $15.00 \%$ & $14 \mathrm{~d}$ & $91.74 \%$ & $-73.18 \%$ & $92.35 \%$ & 0.9717 \\
\hline s & $0.92 * k$ & $10.00 \%$ & $7 \mathrm{~d}$ & $90.87 \%$ & $-50.10 \%$ & $63.78 \%$ & 1.3935 \\
\hline $\mathrm{s}$ & $0.94^{*} \mathrm{k}$ & $5.00 \%$ & $7 \mathrm{~d}$ & $89.76 \%$ & $-57.32 \%$ & $58.10 \%$ & 1.5105 \\
\hline s & $0.9 * \mathrm{k}$ & $20.00 \%$ & $14 \mathrm{~d}$ & $89.76 \%$ & $-78.96 \%$ & $66.52 \%$ & 1.3192 \\
\hline $1.02 * \mathrm{~s}$ & $0.92^{*} \mathrm{k}$ & $5.00 \%$ & $30 \mathrm{~d}$ & $88.73 \%$ & $-54.13 \%$ & $67.63 \%$ & 1.2824 \\
\hline $1.02 *_{\mathrm{s}}$ & $0.9^{*} \mathrm{k}$ & $5.00 \%$ & $30 \mathrm{~d}$ & $88.32 \%$ & $-45.92 \%$ & $56.66 \%$ & 1.5235 \\
\hline $1.02{ }^{*} \mathrm{~s}$ & $0.9^{*} \mathrm{k}$ & $10.00 \%$ & $30 \mathrm{~d}$ & $88.20 \%$ & $-51.46 \%$ & $64.30 \%$ & 1.3406 \\
\hline $\mathrm{s}$ & $0.96 * \mathrm{k}$ & $15.00 \%$ & $14 \mathrm{~d}$ & $86.39 \%$ & $-87.83 \%$ & $96.35 \%$ & 0.8759 \\
\hline s & $0.92^{*} \mathrm{k}$ & $5.00 \%$ & $7 \mathrm{~d}$ & $86.39 \%$ & $-47.58 \%$ & $50.22 \%$ & 1.6804 \\
\hline s & $0.94 * k$ & $20.00 \%$ & $14 \mathrm{~d}$ & $85.41 \%$ & $-82.21 \%$ & $88.68 \%$ & 0.9406 \\
\hline s & $0.94 * k$ & $10.00 \%$ & $7 \mathrm{~d}$ & $85.20 \%$ & $-64.80 \%$ & $74.97 \%$ & 1.1097 \\
\hline
\end{tabular}




\section{Real-data backtests for the Lambda strategies}

As was already pointed out earlier, variants as well as combinations of such Lambda strategies in real trades since summer 2012 have yielded exceptionally good results. In the following we are going to start a first comprehensive study of this type of strategies.

Just like we did for the put-write strategy in the previous section, we are now going to describe the detailed settings and execution of the Lambda strategy, and of the backtesting program on our website. The basic control parameters to be set by the user are

- the available investment capital $I$ at the beginning of each period

- the duration of each period

- the period to be checked (start and end date)

- the type of hedging with long options. Available variants are

- "Put" - hedging against underlying price drops with a single long put option

- "Call" - hedging against underlying price increases with a single long call option

- "Both - left side" - hedging against strong fluctuations of the underlying in any direction, while the maximum-loss parameter described below applies only to underlying price drops

- "Both - right side" - the same principle is applied, but the "max loss" only applies to underlying price increases

- "None" - no long options will be used

- "Dynamic" - the type of long options chosen is decided for each trading period individually according to a formula described below

- the maximum loss as a percentage of the current investment capital, but applied only to the side specified in the type of hedging chosen

- the intensity of the strategy, with a parameter to specify how many option combinations are to be traded, depending on the currently available capital at the beginning of each period, as described in the relevant section below.

In addition to the basic parameters described above, users can provide additional parameters like bid-ask spreads or transaction costs to consider real-market frictions.

\section{Basic parameter settings and execution of the strategy}

The following steps are repeated in a loop starting at the given start date until the end date is reached.

\section{Determining the upcoming trading period}

The program tries to find suitable options so that the actual period length is greater than or equal to and in any case as close as possible to the chosen period length. If no options with that quote date (which satisfy the requirements of the chosen strategy parameters) can be found, so that the actual period length is smaller than the chosen length plus one week, the quote date is moved one day forward, and the process is repeated. 


\section{Choice of short option strikes}

As described in the introduction above, the short put and call options are always chosen with a strike as close as possible to the current underlying price.

\section{Choice of long option strikes}

If only one long option is chosen (or determined by the "dynamic" formula) as the hedging type, the strike is chosen as far as possible from the money, but so that the maximum loss in that direction matches the corresponding parameter.

If one of the "Both" variants is chosen, the same principle is applied for the specified maximum-loss side, while already keeping in mind the price for an additional long option of the other respective type of the same price. This other long option is then chosen as close as possible to the money, but with a price less than or equal to its counterpart.

We want to illustrate this using a real data example. We consider the 14-day period from February 14 until February 28, 2018. We went short on a contract (100 units) of put options with price $P_{S}=33.45$ USD and a contract of call options with price $C_{S}=30.80$ USD, both with a strike $K_{S}$ of 2700 . The maximum loss is set at $3 \%$. As we start with a capital of 100,000 USD in this example, the absolute maximum loss $M$ accounts for 30 USD per unit, i.e., 3,000 USD per contract. (Note that choosing a negative maximal loss as a parameter value is also possible. For example, a maximal loss of $-2 \%$ means a minimal profit of $2 \%$.) Let us now take a look at the choice of long options for the respective settings in the "long type" section:

- "put" setting: The single long put with ask price $P_{L}$ and strike $K_{L}$ is chosen according to the formula $K_{L}-K_{S}+P_{S}+C_{S}-P_{L} \geq M$, which is equivalent to $P_{L} \leq K_{L}-K_{S}+P_{S}+C_{S}-M$. So we look for the put option with the lowest possible strike that meets this condition, and get one with $P_{L}=12.7$ USD and $K_{L}=2620$, of which we buy one contract.

- "call" setting: We search for a long call with ask price $C_{L}$ and the highest possible strike $K_{L}$ in the same manner, but with the adapted condition $C_{L} \leq-K_{L}+K_{S}+C_{S}+P_{S}-M$, and get an option with $C_{L}=4.2$ USD and $K_{L}=2790$.

- With the "both - left side" setting, a put option is chosen in basically the same way as for the "put" setting, except that the formula already accounts for a call option of the same price. Therefore, the condition $P_{L} \leq K_{L}-K_{S}$ $+C_{S}+P_{S}-M-P_{L}$ returns a put option with $P_{L}=16$ USD and $K_{L}=2640$. Then, in order to also hedge the right side of the profit function, we look for a call option with the lowest possible strike so that $C_{L} \leq P_{L}$, which in our case turns out to be a call option with price $C_{L}=15.4$ USD and strike $K_{L}=2735$. We buy one contract of each.

- The same principle is applied for the setting "both - right side". The call option with the highest possible strike needs to meet the condition $C_{L} \leq-K_{L}+K_{S}+C_{S}+P_{S}-M-C_{L}$ while $P_{L} \leq C_{L}$ needs to hold for the put. We get a call option with $C_{L}=5.3$ USD and strike 2780 and a put option with $P_{L}=5.2$ USD and strike 2540 . 


\section{Dynamic mode}

If the setting "dynamic" is chosen in the hedging type section, the choice of long option types is determined dynamically at the beginning of each period, based on the maximum ( $\max$ ) and minimum ( $\min$ ) underlying closing price on the last 5 trading days and the current underlying price $s$, according to the following principle:

- If $\max >1.02 \cdot s$ and $\min >0.995 \cdot s$, the "call" setting is chosen for this period

- If $\max <1.005 \cdot s$ and $\min <0.98 \cdot s$, the "put" setting is chosen for this period

- Otherwise, the setting "none" is chosen for this period

To illustrate this, we look at the three consecutive trade periods starting on 14 February 2018. At the beginning of each period, we examine the underlying closing values of the last trading days.

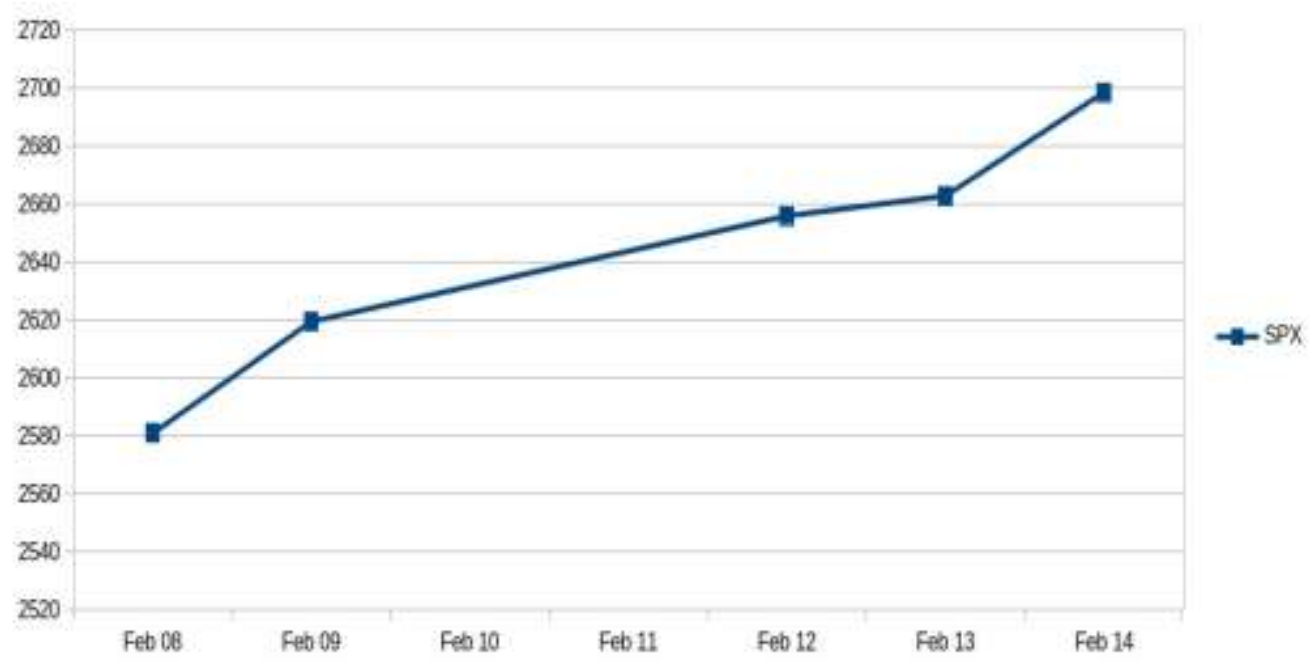

Figure 15. Last trading days before February 14

We see that the maximum underlying value in this interval amounts to 2698.63 and equals the current underlying price at the beginning of the period, while the minimum value is 2581 . Therefore, since $2698.63<1.005 \cdot 2698.63$ and $2581<0.98 \cdot 2698.63$, according to the above formula, we choose the "Put" setting for the period between February 14 and February 28.

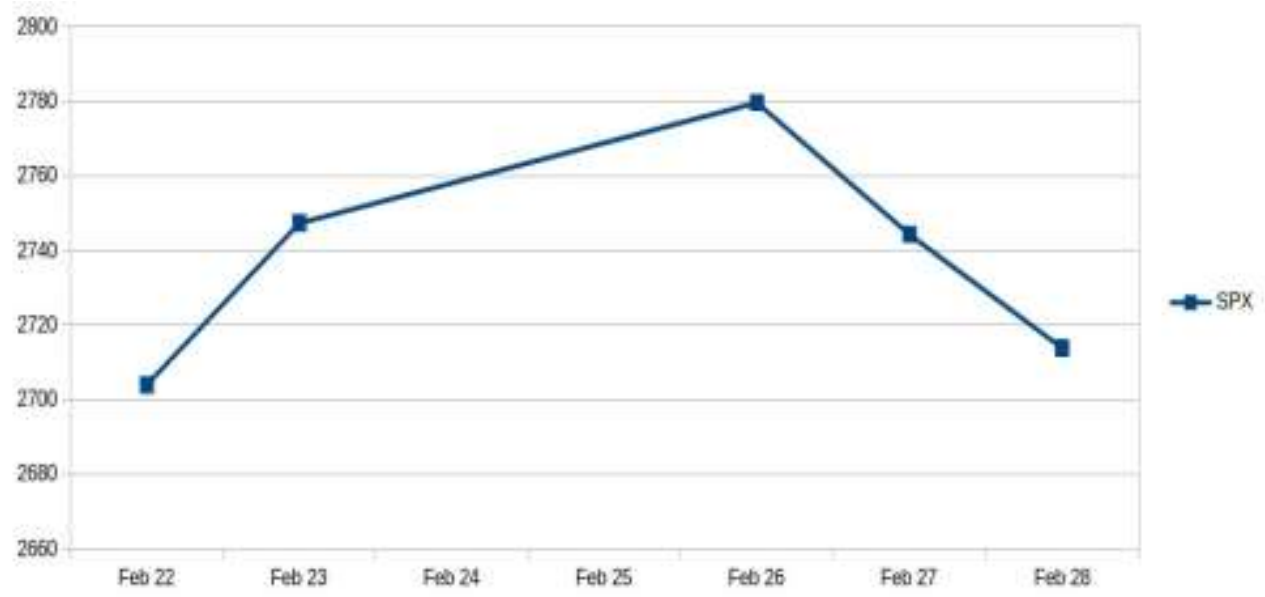

Figure 16. Last trading days before February 28 
On February 28, as the graphic shows, the current underlying value held at 2713.83 while in the last trading days before that, the maximum was 2779.60 and the minimum was 2703.96. As $2779.60>1.02 .2713 .83$ and $2703.96>$ $0.995 \cdot 2713.83$, we choose the "Call" setting for the period between February 28 and March 14.

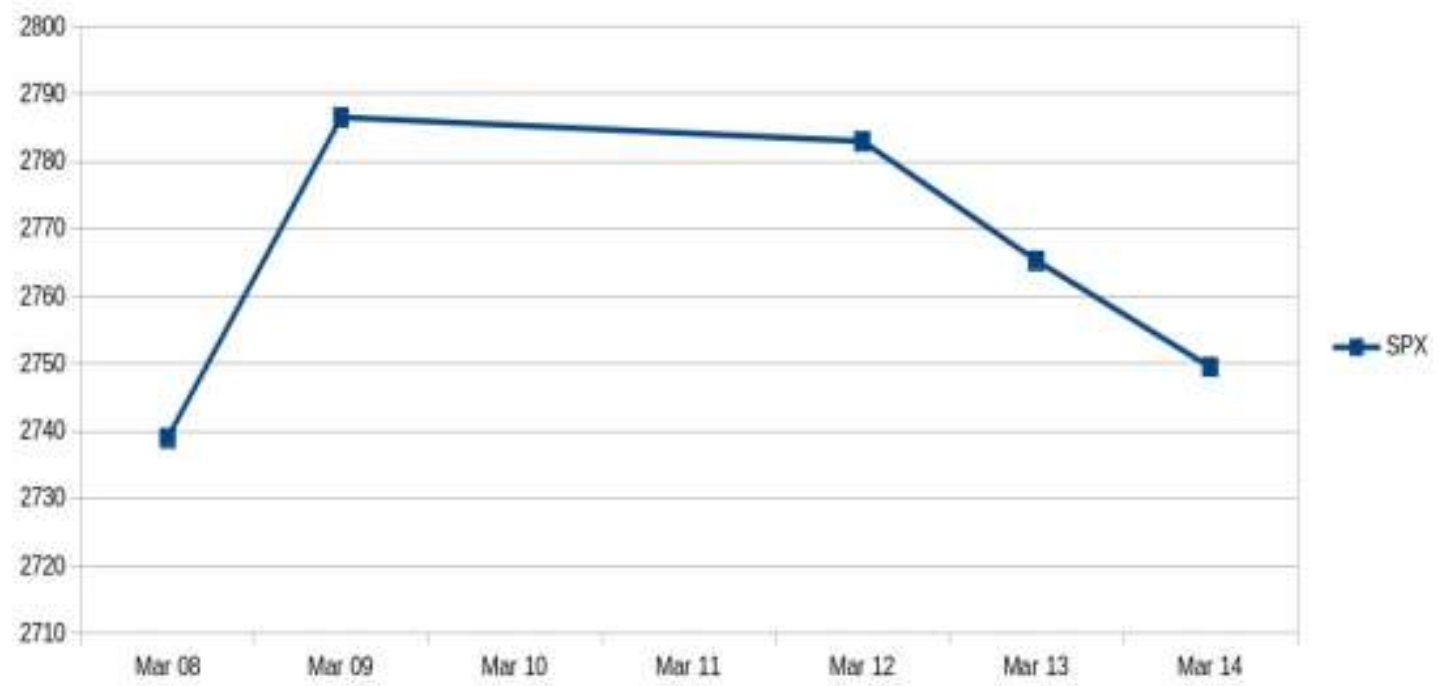

Figure 17. Last trading days before March 14

As we can easily observe, none of the two conditions (for buying any long position) holds for the last trading days before the next period, where the maximum underlying value amounted to 2786.57 , the minimum to 2738.97 and the current value on March 14 was 2749.48. We consequently choose the setting "none" and hence don't acquire any long options for the period between March 14 and March 28.

The consideration on which this strategy is based is essentially a "short-term mean reversion assumption". The user of this approach believes in mean reversion after a sudden strong decline or increase of the index, and hence wants to hedge against such a mean reversion.

\section{Determining the number of combinations to trade}

At the beginning of each period, the program determines the number of option combinations $n$ to trade, depending on the currently available capital $I$ and the intensity parameter $i$. A combination is a set of two short option contracts and 0 to 2 long option contracts, as defined in the strategy settings. The number of combinations is determined according to the formula

$$
n=\left\lceil\frac{I \cdot i}{100000}\right\rceil
$$

\section{Exiting the strategy}

The program will regularly terminate its strategy execution if the start date of the next period is later than the designated strategy's end date minus the specified period length.

In addition, it will exit the strategy if the capital available at the beginning of a period is less than 50,000 USD. 


\section{Additional Parameters}

As in the program for the put-write strategy, users can also provide additional parameters to take existing market restrictions into account.

\section{Transaction costs}

The transaction costs per contract can be provided and are subtracted accordingly.

\section{Bid-ask spread}

The market buying price $(m b p)$ of an option is determined based on its bid and ask prices as well as the specified value of the bid-ask spread parameter as follows:

- For buying options we use the price:

$$
m b p=\frac{b i d+a s k}{2}+\text { spread }
$$

- For selling options we use the price:

$$
m b p=\frac{b i d+a s k}{2}-s p r e a d
$$

Overview of results for Lambda strategies in the period from 2012 to 2020

In this section, we will present a general overview of representative results of the execution of the Lambda strategy backtests between summer 2012 and summer 2020 .

The following settings applied to all of the strategy executions which we have carried out:

- Initial investment capital: 100,000 USD

- Start date: July 1, 2012

- End date: July 31, 2020

- Transaction costs per contract: 5 USD

- Bid-ask spread: 0.1

- Intensity: 1

We have carried out backtesting for a wide range of combinations of fixed parameter choices. Detailed results of the backtests, and all the details of each strategy's performance in the time period from 2012 until 2020 can be found on our website. In addition, any choice of parameters can be tested using the testing program on our website.

Compared with the results that the second author and his trading team obtained with these strategies in real-life trading during this time period and compared also with the results of the put-write strategies, the backtesting results for the Lambda strategies are rather poor. In Table 10 we show the 10 best strategies in our tests with respect to annual return. In Table 11 we show the 10 best strategies in our tests with respect to Sharpe ratio. 
Table 10. Best strategies between July 2012 and August 2020 in terms of return per annum

\begin{tabular}{rlr|rrrr}
\hline Period (days) & Long type & Max loss & Return p.a. & Max drawdown & Volatility p.a. & Sharpe \\
\hline 7 & call & $-1.50 \%$ & $22.54 \%$ & $-54.14 \%$ & $44.19 \%$ & 0.4875 \\
7 & call & $-2.00 \%$ & $18.17 \%$ & $-59.09 \%$ & $48.81 \%$ & 0.3517 \\
3 & call & $-2.00 \%$ & $16.93 \%$ & $-55.17 \%$ & $60.92 \%$ & 0.2614 \\
30 & call & $-1.50 \%$ & $10.99 \%$ & $-61.84 \%$ & $30.56 \%$ & 0.3270 \\
30 & call & $-0.50 \%$ & $9.90 \%$ & $-54.81 \%$ & $27.73 \%$ & 0.3210 \\
30 & call & $-1.00 \%$ & $9.85 \%$ & $-62.35 \%$ & $29.95 \%$ & 0.2955 \\
7 & both-right & $-0.50 \%$ & $8.53 \%$ & $-7.97 \%$ & $35.81 \%$ & 0.2103 \\
30 & call & $0.00 \%$ & $7.96 \%$ & $-59.78 \%$ & $31.18 \%$ & 0.2232 \\
14 & call & $-0.50 \%$ & $7.46 \%$ & $-69.17 \%$ & $28.36 \%$ & 0.2278 \\
14 & both-right & $-0.50 \%$ & $6.94 \%$ & $-7.89 \%$ & $17.26 \%$ & 0.3444 \\
\hline
\end{tabular}

Table 11. Best strategies between July 2012 and August 2020 in terms of Sharpe ratio

\begin{tabular}{rlr|rrrr}
\hline period (days) & long type & max loss & return p.a. & max drawdown & volatility p.a. & Sharpe \\
\hline 7 & call & $-1.50 \%$ & $22.54 \%$ & $-54.14 \%$ & $44.19 \%$ & 0.4875 \\
30 & both-right & $0.00 \%$ & $5.57 \%$ & $-14.37 \%$ & $9.90 \%$ & 0.4619 \\
7 & call & $-2.00 \%$ & $18.17 \%$ & $-59.09 \%$ & $48.81 \%$ & 0.3517 \\
14 & both-right & $-0.50 \%$ & $6.94 \%$ & $-7.89 \%$ & $17.26 \%$ & 0.3444 \\
30 & call & $-1.50 \%$ & $10.99 \%$ & $-61.84 \%$ & $30.56 \%$ & 0.3270 \\
30 & call & $-0.50 \%$ & $9.90 \%$ & $-54.81 \%$ & $27.73 \%$ & 0.3210 \\
30 & call & $-1.00 \%$ & $9.85 \%$ & $-62.35 \%$ & $29.95 \%$ & 0.2955 \\
7 & both-right & $0.00 \%$ & $4.68 \%$ & $-38.21 \%$ & $12.74 \%$ & 0.2888 \\
3 & call & $-2.00 \%$ & $16.93 \%$ & $-55.71 \%$ & $60.92 \%$ & 0.2614 \\
14 & call & $-0.50 \%$ & $7.46 \%$ & $-69.17 \%$ & $28.36 \%$ & 0.2278 \\
\hline
\end{tabular}

Only a few of the tested strategies show an outperformance over the S\&P500 in the same time period. The S\&P500 had an annual return of $11.41 \%$, a Sharpe ratio of 0.614 and a maximum drawdown of $-33.92 \%$ (from February 19 , 2020 to March 23, 2020) in this time period.

How can we explain this obvious discrepancy between the performance of real-trade Lambda strategies (especially when carried out with short-term options (3d until 7d)) and the backtests? 
Our most likely explanation for this is based on the flexibility of the parameter choices based on the current market scenario when executing the Lambda strategies in real trading. We did not use an a priori defined choice of parameters but tried to "optimally" adapt the choice of parameters for each new period to the given circumstances of the S\&P500 market.

However, it is hard to efficiently optimize dynamic choices of optimal parameters based on given market scenarios. Further research based on these observations is called for. A first (and of course too simple and insufficient) attempt toward an automated adapted choice of parameters was made with the above described "dynamic mode". Going forward, AI and machine-learning techniques should prove helpful in this regard and should be investigated with a view to optimizing such adapted parameter choices. Maybe modeling of different market scenarios and executing these strategies on the models also provides insight into different approaches given a specific market environment.

In conclusion we want to highlight two of the more successful parameter choices.

- The strategy "7 days, call, $-1.5 \%$ maximum loss" is the Lambda strategy with the highest annual return in the period from 2012 to July 2020.

- The strategy "7 days, both-right, $-0.5 \%$ maximum loss" is a strategy with a stable, clearly positive return, and with a positive return in all the relevant - including the critical - sub-periods, i.e., in the period from 2012 to 2017, in 2018, from 2012 to 2019, and in the period from January to July 2020. Moreover, it shows only a moderate maximum drawdown.

In Table 12 we see the most relevant performance data for these two strategies, and in Figure 14 we illustrate the performance of these two strategies in comparison with the performance of the S\&P500 index in the period from 2012 to July 2020. Again, all the details of every single trade of these (and all the other strategies) can be found on our website.

Table 12. Most relevant performance data for two strategies

\begin{tabular}{|c|c|c|c|c|c|c|c|c|}
\hline strategy & 7 days, & call & $-1.5 \%$ & $\max \operatorname{loss}$ & 7 days, & both right, & $-0.5 \%$ & $\max$ loss \\
\hline return p.a. & & & & $22.54 \%$ & & & & $8.53 \%$ \\
\hline return & & & & $408.49 \%$ & & & & $92.48 \%$ \\
\hline return until end 2017 & & & & $223.73 \%$ & & & & $12.62 \%$ \\
\hline return 2018 & & & & $-27.54 \%$ & & & & $28.71 \%$ \\
\hline return until end 2019 & & & & $321.63 \%$ & & & & $52.56 \%$ \\
\hline return 2020 & & & & $20.60 \%$ & & & & $26.17 \%$ \\
\hline max drawdown & & & & $-54.14 \%$ & & & & $-7.97 \%$ \\
\hline volatility p.a. & & & & $44.19 \%$ & & & & $25.81 \%$ \\
\hline Sharpe & & & & 0.4875 & & & & 0.2103 \\
\hline
\end{tabular}




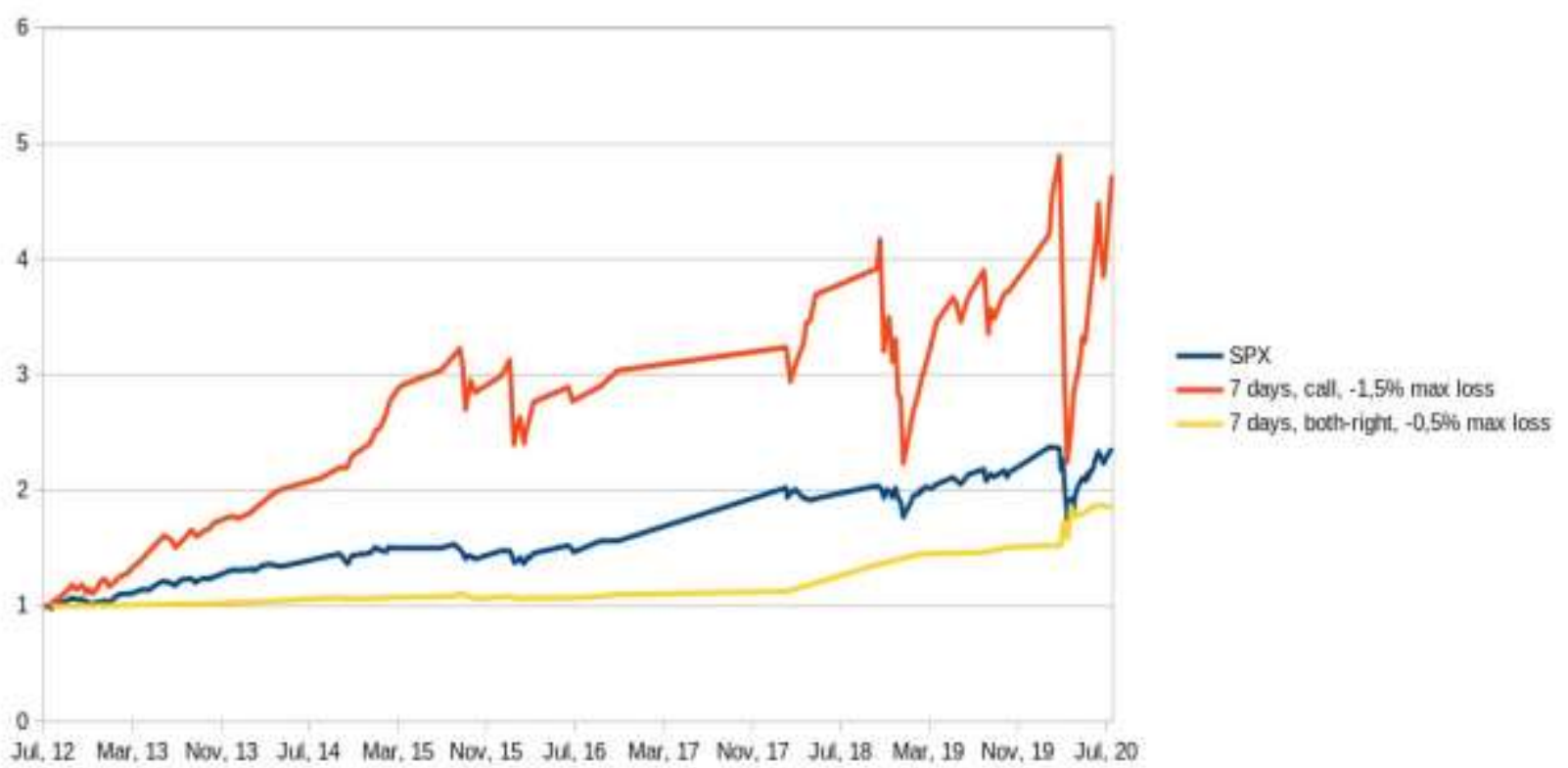

Figure 18. Performance of two strategies in comparison with the S\&P500

\section{Summary}

Our analysis of S\&P500 put-write strategies for the period from 2010 to 2020 show that these strategies yield a clear outperformance for a wide choice of parameter values, and they confirm results of investigations into the performance of such strategies that were given for the period from 1990 to 2010 in (Larcher, Del Chicca, and Szölgyenyi, 2013). We show - based on simulation experiments - that to a certain extent, this outperformance can be explained by a negative correlation between the S\&P500 index and its volatility index VIX.

In contrast to this, the backtests of the so-called Lambda strategies generally do not show a satisfying performance for the time period from 2012 until 2020, despite the fact that real-life trading in this time period provided very positive and stable results. One main reason for this seeming discrepancy between theoretical and real trading results most probably lies in the adaptability of the strategy's parameters to different market conditions in the real trades, whereas in the backtests we worked with strictly pre-defined parameter choices only. This calls for further investigation with more flexible parameter choices dependent on given market scenarios.

We provide free backtesting software and detailed data on all of our simulation and backtesting results on our website at www.lsqf.org.

\section{Acknowledgements}

The authors wish to thank an anonymous reviewer very much for thoroughly reading this paper and for many important remarks and valuable suggestions! The authors are supported by the Austrian Science Fund (FWF), Project F5507-N26, which is part of the Special Research Program Quasi-Monte Carlo Methods: Theory and Applications, and by the Land Upper Austria research funding. 


\section{References}

Bakshi, Gurdip and Nikunj Kapadia (2003). „Delta-Hedged Gains and the Negative Market Volatility Risk

Premium“. In: Review of Financial Studies 16.2, pp. 527-566.

Day, Theodore E. and Craig M. Lewis (1997). „Initial margin policy and stochastic volatility in the crude oil futures market“. In: The review of financial studies.

Del Chicca, Lucia and Gerhard Larcher (2012). „A Comparison of Different Families of Put-Write Option

Strategies“. EN. In: ACRN Journal of Finance and Risk Perspectives 1.1, pp. 1-14.

French, Kenneth R., G.William Schwert, and Robert F. Stambaugh (1987). „Expected stock returns and volatility“. In: Journal of Financial Economics 19.1, pp. 3-29. issn: 0304-405X. doi: https://doi.org/

10.1016/0304-405X(87)90026-2.

Jackwerth, Jens Carsten and Mark Rubinstein (1996). „Recovering Probability Distributions from Option Prices“. In: The Journal of Finance 51.5, pp. 1611-1631. issn: 00221082, 15406261. url: http://www. jstor.org/stable/2329531.

Koopman, Siem Jan, Borus Jungbacker, and Eugenie Hol (2005). „Forecasting daily variability of the S\&P 100 stock index using historical, realised and implied volatility measurements“. In: Journal of Empirical

Finance 12.3, pp. 445-475. issn: 0927-5398. doi: https://doi.org/10.1016/j.jempfin.2004.04.009.

Larcher, Gerhard (2020). Quantitative Finance: Strategien, Investments, Analysen. 1. Auflage 2020. Wiesbaden:

Springer Fachmedien Wiesbaden GmbH and Springer Gabler. isbn: 9783658291570.

Larcher, Gerhard, Lucia Del Chicca, and Michaela Szölgyenyi (2013). „Modeling and Performance of Certain Put-WriteStrategies“. In: The Journal of Alternative Investments 15.4, pp. 74-86. issn: 1520-3255. doi:

10.3905/jai.2013.15.4.074.

Santa-Clara, Pedro and Alessio Saretto (2009). „Option strategies: Good deals and margin calls“. In: Journal of financial markets 12.3, pp. 391-417.

Ungar, Jason and Matthew T. Moran (2009). „The Cash-secured PutWrite Strategy and Performance of Related Benchmark Indexes“. In: The Journal of Alternative Investments 11.4, pp. 43-56. issn: 15203255. doi: 10.3905/JAI.2009.11.4.043.

(C) 2021 by the authors. Licensee ACRN Publishing, Austria, Editor in Chief Prof. Dr. Othmar M. Lehner. This article is an open access article distributed under the terms and conditions of the Creative Commons Attribution (CC BY SA) license (https://creativecommons.org/licenses/by-sa/4.0/) 